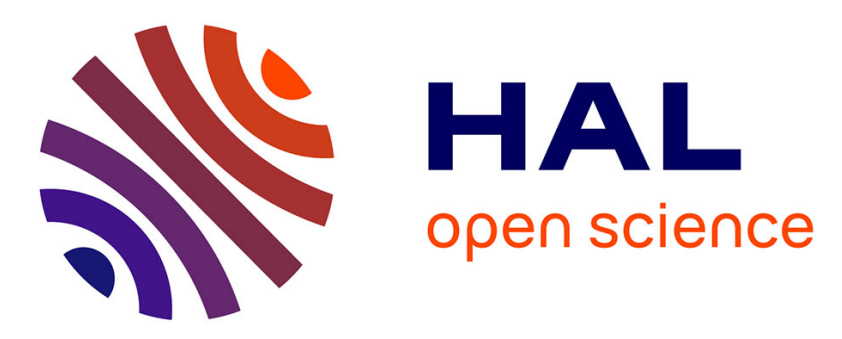

\title{
Observer-based control of linear complementarity systems
}

\author{
W. P. Maurice H. Heemels, M. Kanat Camlibel, Johannes M. Schumacher, \\ Bernard Brogliato
}

\section{- To cite this version:}

W. P. Maurice H. Heemels, M. Kanat Camlibel, Johannes M. Schumacher, Bernard Brogliato. Observer-based control of linear complementarity systems. International Journal of Robust and Nonlinear Control, 2011, Special Issue: New Directions on Hybrid Control Systems, 21 (10), pp.1193-1218. 10.1002/rnc.1626 . hal-00756195

\section{HAL Id: hal-00756195 \\ https://hal.inria.fr/hal-00756195}

Submitted on 27 Nov 2012

HAL is a multi-disciplinary open access archive for the deposit and dissemination of scientific research documents, whether they are published or not. The documents may come from teaching and research institutions in France or abroad, or from public or private research centers.
L'archive ouverte pluridisciplinaire HAL, est destinée au dépôt et à la diffusion de documents scientifiques de niveau recherche, publiés ou non, émanant des établissements d'enseignement et de recherche français ou étrangers, des laboratoires publics ou privés. 


\title{
Observer-based control of linear complementarity systems
}

\author{
W.P.M.H. Heemels ${ }^{1, *}$, M.K. Camlibel $^{2}$, J.M. Schumacher ${ }^{3}$, and B. Brogliato ${ }^{4}$ \\ ${ }^{1}$ Department of Mechanical Engineering, Eindhoven University of Technology, The Netherlands \\ 2 Department of Mathematics, University of Groningen, The Netherlands \\ ${ }^{3}$ Department of Econometrics and Operations Research, Tilburg University, The Netherlands \\ ${ }^{4}$ INRIA Rhône-Alpes, France
}

\begin{abstract}
SUMMARY
In this paper we present observer and output-based controller design methods for linear complementarity systems (LCS) employing a passivity approach. Due to various inherent properties of LCS, such as the presence of state jumps, mode dynamics described by differential and algebraic equations (DAEs), and regions for certain modes being lower dimensional, various observer and control design schemes that have been proposed for other classes of (hybrid) dynamical systems do not apply to LCS. In particular, we present an observer design method for LCS which is effective even in the presence of state jumps. We show the well-posedness of the observer, in the sense of existence and uniqueness of solution trajectories for the estimated state, and prove the global exponential stability of the observation error. These two properties guarantee that the estimated state exponentially recovers the state of the system. For the problem of stabilization based on output measurements only, we adopt an observer-based control approach in which we apply a state feedback law to the estimated state obtained from the observer. We prove that the resulting closed-loop system is well-posed and globally exponentially stable. In order to show the well-posedness of the closed loop, novel well-posedness results for LCS based on low-index properties are presented.
\end{abstract}

KEY WORDS: Output feedback, stabilization, observer design, hybrid systems, complementarity systems.

\section{Introduction}

Complementarity systems form a class of hybrid dynamical systems that has received considerable attention in recent years $[8,9,11,12,25,26,42-44,48-50]$. The linear complementarity system $\operatorname{LCS}(A, B, C, D, E, F)$ with $A \in \mathbb{R}^{n \times n}, B \in \mathbb{R}^{n \times m}, C \in \mathbb{R}^{m \times n}, D \in \mathbb{R}^{m \times m}, E \in \mathbb{R}^{n \times p}$ and $F \in \mathbb{R}^{m \times p}$ is given by

$$
\begin{aligned}
\dot{x}(t) & =A x(t)+B w(t)+E u(t) \\
z(t) & =C x(t)+D w(t)+F u(t) \\
0 & \leq z(t) \perp w(t) \geq 0,
\end{aligned}
$$

${ }^{*}$ Correspondence to: Department of Mechanical Engineering, Eindhoven University of Technology, P.O. Box 513, 5600 MB Eindhoven, The Netherlands 
where the inequalities are interpreted componentwise and $\perp$ indicates the orthogonality between the complementarity vectors $z(t) \in \mathbb{R}^{m}$ and $w(t) \in \mathbb{R}^{m}$, i.e, $z^{\top}(t) w(t)=0$. In this description $x(t) \in \mathbb{R}^{n}$ denotes the state variable and $u(t) \in \mathbb{R}^{p}$ is the control input at time $t \in \mathbb{R}_{+}$. The complementarity conditions (1c) constitute a particular set of equalities and inequalities of the type that appears for instance in the Karush-Kuhn-Tucker conditions for optimality under inequality constraints, in the voltage-current relationship for ideal diodes, and in the conditions between unilateral constraints and reaction forces in constrained mechanics. As a result, applications of the dynamic complementarity framework include mechanical systems with unilateral constraints, constrained optimal control problems, and switched electrical circuits.

While the class of LCS has its own typical characteristics, it can be related to other classes of hybrid systems. Indeed, observe that (1c) implies that $w_{i}(t)=0$ or $z_{i}(t)=0$ for all $i \in \bar{m}:=\{1, \ldots, m\}$. As a consequence, the system (1) has $2^{m}$ modes. Each mode can be characterized by the active index set $J \subseteq \bar{m}$, which indicates $z_{i}=0, i \in J$, and $w_{i}=0, i \in J^{c}$, where $J^{c}:=\{i \in \bar{m} \mid i \notin J\}$. For mode $J$ the dynamics is given by the linear differential and algebraic equations (DAEs)

$$
\begin{gathered}
\dot{x}(t)=A x(t)+B w(t)+E u(t), \\
z(t)=C x(t)+D w(t)+F u(t), \\
z_{i}(t)=0, i \in J, \text { and } w_{i}(t)=0, i \in J^{c} .
\end{gathered}
$$

The evolution of system (1) is governed by (2) for mode $J$ as long as the remaining inequalities in (1c)

$$
z_{i}(t) \geq 0, i \in J^{c} \text { and } w_{i}(t) \geq 0, i \in J
$$

are satisfied. Impending violation of (3) triggers a mode change. As a consequence, during the evolution in time of the system several mode dynamics are active successively. This indicates that LCS might be recast within the hybrid automaton framework [7,32,33]. However, with exception only of the very simplest cases, the reformulation of LCS dynamics into the hybrid automaton framework leads to voluminous and opaque system descriptions as all the mode dynamics, invariants, guards and reset relations would have to be enumerated. This effect is already evident in the example worked out in [22], which concerns an electrical circuit with two diodes. Alternatively one can rewrite LCS in the formulation $\dot{x} \in \mathcal{F}(x)$ for $x \in \mathcal{C}$ and $x^{+} \in \mathcal{G}(x)$ for $x \in \mathcal{D}$, where $\mathcal{F}$ and $\mathcal{G}$ are setvalued maps from $\mathbb{R}^{n}$ to $\mathbb{R}^{n}$ and $\mathcal{C}$ and $\mathcal{D}$ are subsets of $\mathbb{R}^{n}$, as advocated in [17, 18,41]. Again, the transformation is in general cumbersome, and the resulting system data $\mathcal{F}, \mathcal{G}, \mathcal{C}$ and $\mathcal{D}$ do generally not satisfy the assumptions adopted in $[17,18,41]$ (cf. the example below). Structural properties become harder to study and compactness of descriptions is lost when LCS are translated to such generic frameworks.

To illustrate the links between LCS and other (sub)classes of hybrid dynamical systems, let us consider an LCS (1) with a single complementarity pair and $F=0$ :

$$
\dot{x}=A x+b w+e u ; z=c^{\top} x+d w ; 0 \leq z \perp w \geq 0,
$$

where $A \in \mathbb{R}^{n \times n}, b \in \mathbb{R}^{n \times 1}, c \in \mathbb{R}^{n \times 1}, d \in \mathbb{R}$, and $0 \neq e \in \mathbb{R}^{n \times 1}$. As either $z=0$ or $w=0$, this system has two modes. When $d>0$, one can rewrite (4) as

$$
\dot{x}= \begin{cases}A x+e u & \text { if } c^{\top} x \geq 0 \\ \left(A-b d^{-1} c^{\top}\right) x+e u & \text { if } c^{\top} x \leq 0,\end{cases}
$$

which is a piecewise linear (PWL) system $[28,45]$. When $d=0$ and $c^{\top} b>0$, we have

$$
\dot{x}= \begin{cases}A x+e u & \text { if }\left(c^{\top} x>0\right) \text { or }\left(c^{\top} x=0 \text { and } c^{\top} A x+c^{\top} e u \geq 0\right) \\ P(A x+e u) & \text { if } c^{\top} x=0 \text { and } c^{\top} A x+c^{\top} e u<0,\end{cases}
$$


where $P=I-b\left(c^{\top} b\right)^{-1} c^{\top}$. In this case one has a bimodal PWL system as well, but the second subsystem 'lives' on a lower dimensional subspace given by $c^{\top} x=0$, which is a situation that has received little attention in the literature on PWL systems. Since the system equations do not allow the state to enter the region given by $c^{\top} x<0$, state jumps must occur. Another observation is that in the case when $d=c^{\top} b=0, c^{\top} A b>0$ and $e=0$ (no external inputs), the flow set, i.e. the set of states from which the system can continue with a smooth solution at least for some time, is given by all $x_{0}$ such that $\left(c^{\top} x_{0}, c^{\top} A x_{0}\right) \succeq 0$ (see [25, Thm. 6.8]), where $\succeq$ denotes the lexicographic ordering. This indicates that the flow set has no simple closedness properties.

The above observations show that although LCS have connections to PWL systems and other classes of hybrid systems, they also have their own peculiarities. For instance, due to the presence of state jumps (think of impacts in constrained mechanical systems), continuous-time LCS are differentiated from much of the work done for continuous-time PWL systems. Although for discrete-time LCS strong equivalence links have been established in [23] with piecewise affine systems [45] and other classes of hybrid models such as min-max-plus-scaling systems [14] and mixed logic dynamic systems [6], in the continuous-time framework, which is the natural habitat for most of the LCS applications, such broad equivalence relations are out of the question. There are relations though of LCS to other specific classes of nonsmooth systems such as projected dynamical systems $[9,15,26,36]$ and "normal-cone differential inclusions (DIs)." Indeed, LCS may be considered as DIs, whose set values are normal cones to (possibly time-varying) convex sets as shown in e.g. $[9,26]$. However, the DIs that are obtained in this way generally do not satisfy the boundedness and upper semicontinuity conditions that are commonly used in the literature on DIs. Such evolution problems belong to the class of measure DIs and they require specific mathematical and numerical analysis tools, see, e.g., $[1,34,35]$.

The attention that LCS received recently is not surprising given the broad range of applications. Several fundamental system-theoretic issues such as well-posedness, discretization (simulation), controllability, observability, stabilizability and stability were studied in the papers already cited at the beginning of this section. In this paper the emphasis is on stabilization by dynamic output feedback, a subject that is central in control theory but that has received little attention so far in the context of LCS with the exception of the conference paper [20] which announced (without proofs) some of the results in the present paper. Our approach is based on the classical separation method, which in the linear systems case works as follows (see for instance [30, §5.2]). Given a system

$$
\dot{x}(t)=A x(t)+E u(t), \quad y(t)=G x(t),
$$

define an observer by

$$
\dot{\hat{x}}(t)=A \hat{x}(t)+E u(t)+L(y(t)-G \hat{x}(t))
$$

and set $u(t)=K \hat{x}(t)$. Define $e(t)=x(t)-\hat{x}(t)$. The joint system of state and error dynamics is

$$
\left[\begin{array}{c}
\dot{x}(t) \\
\dot{e}(t)
\end{array}\right]=\left[\begin{array}{cc}
A+E K & -E K \\
0 & A-L G
\end{array}\right]\left[\begin{array}{l}
x(t) \\
e(t)
\end{array}\right],
$$

which shows that the closed-loop system is stable if the design matrices $K$ and $L$ are such that both $A+E K$ and $A-L G$ are stable. This approach is not readily extended to nonlinear systems or hybrid systems in general. We show in this paper that the separation technique can however be applied to LCS. A qualification that needs to be made here is that our design conditions are more stringent than in the linear systems case; we require passivity rather than just stability. While in the linear systems case the well-posedness (existence and uniqueness of solutions) of the closed-loop system is automatically ensured, in the hybrid case the well-posedness of the closed-loop system is in fact a major concern. This 
may give some reason to expect that stronger conditions would indeed be needed. Although passivity appears in our design conditions, we do not require passivity properties for the closed-loop system as a whole, and our stability and well-posedness arguments are based on a mixture of passivity and "low-index" arguments. The low-index arguments extend earlier passivity-based well-posedness results $[11,21,24]$. In addition to these new results on well-posedness, we also address the observer design problem for LCS. Here one needs to consider various issues that do not occur in the smooth (singlemode) case. First of all, one has to face the complication that the controlled system and the observer may not always be in the same mode. Secondly, the presence of jumps in the state variable is known to cause major complications for observer design in the general hybrid systems case. The contributions of this paper can therefore be summarized as follows:

(i) New well-posedness results for LCS using low-index conditions.

(ii) Observer design methods for LCS.

(iii) Observer-based controllers that result in a well-posed and globally exponentially stable closedloop system using a separation method.

Several papers are available on observer design for hybrid systems, especially in the context of switched and piecewise linear systems, e.g. [2, 27, 29, 38, 39,47]. These results do not apply to LCS however, as LCS typically exhibit lower dimensional regions and state jumps, as mentioned before. Observer and observer-based controller design methods for Lur'e type systems as studied in $[3,5,16,40]$ are also related to LCS. Indeed, one can consider LCS as a kind of Lur'e type system in which the linear system (1a)-(1b) is interconnected with the non-smooth and unbounded complementarity relations in (1c). Typically, the results in $[3,5,16,40]$ study locally Lipschitz slope restricted nonlinearities in the feedback path. As such their conditions do not allow for the nonsmoothness and set-valued nonlinearities (and even state jumps) as induced by the complementarity relations. Observer designs for differential inclusions with bounded set-values are treated in [37]. Since complementarity conditions are unbounded, LCS are not covered in the framework of [37]. In [10] observer design is considered for a class of Lur'e type systems with multivalued mappings in the feedback path. This class contains a subset of LCS as a special case. However, [10] requires that in (1) $D=0$, which excludes various LCS of practical interest in the area of power converters, see e.g. [50]. Moreover, both [37] and [10] consider continuous solution trajectories only, which do not allow for systems with state jumps. In summary, while there are various interesting approaches for hybrid observer design, none of them includes all the peculiarities of LCS.

The outline of the paper is as follows. Before focussing on controller and observer design for LCS, we explain the solution concepts for LCS and also provide novel well-posedness (existence and uniqueness of solutions) results for LCS under weaker assumptions than in earlier work. We obtain global existence results that exclude the Zeno phenomenon of livelock (an infinite number of discrete actions at the same time instant) and which provide continuations beyond accumulation points of mode switching times. Next, we present passivity-based conditions for state feedback design and observer design for LCS. In particular, this means that we present methods for observer design for systems without knowing the mode and while allowing for state resets. Based on the separate designs of the state feedback and the observer, we show that the interconnection of the observer and the state feedback results in a closed-loop system that is well-posed and globally exponentially stable. Finally, we state the main conclusions of this paper and an outlook for future research. 


\section{Preliminaries}

The symbol $\mathbb{R}$ denotes the set of real numbers, $\mathbb{R}_{+}:=[0, \infty)$ the nonnegative real numbers, $\mathcal{L}_{2}(\mathbb{T})$ the square integrable functions on a time-interval $\mathbb{T} \subseteq \mathbb{R}$, and $\mathcal{B}$ the Bohl functions (i.e. functions having strictly proper rational Laplace transforms) defined on $\mathbb{R}_{+}$. Note that sines, cosines, exponentials, polynomials and their sums and products are all Bohl functions. The distribution $\delta_{t}^{(i)}$ stands for the $i$-th distributional derivative of the Dirac impulse supported at $t$. The dual cone of a set $\mathcal{Q} \subseteq \mathbb{R}^{n}$ is defined by $\mathcal{Q}^{*}=\left\{x \in \mathbb{R}^{n} \mid x^{\top} y \geq 0\right.$ for all $\left.y \in \mathcal{Q}\right\}$. For a positive integer $m$, the set $\bar{m}$ is defined as $\{1,2, \ldots, m\}$ and $2^{\bar{m}}$ denotes the collection of all subsets of $\bar{m}$. A vector $u \in \mathbb{R}^{k}$ is called nonnegative, denoted by $u \geq 0$, if $u_{i} \geq 0$ for all $i \in \bar{k}$. This means that inequalities for vectors are interpreted componentwise. The orthogonality $u^{\top} y=0$ between two vectors $u \in \mathbb{R}^{k}$ and $y \in \mathbb{R}^{k}$ is denoted by $u \perp y$. As usual, we say that $(A, B, C)$ (or sometimes $(A, B, C, D)$ ) is minimal, when the matrices $\left[B A B \ldots A^{n-1} B\right]$ and $\left[C^{\top} A^{\top} C^{\top} \ldots\left(A^{\top}\right)^{n-1} C^{\top}\right]$ have full rank. A (not necessarily symmetric) matrix $M \in \mathbb{R}^{k \times k}$ is called positive definite if $x^{\top} M x>0$ for all $x \neq 0$ and we write $M>0$. It is called nonnegative definite, if $x^{\top} M x \geq 0$ for all $x \in \mathbb{R}^{k}$ and we write $M \geq 0$. For a matrix $M \in \mathbb{R}^{k \times l}$ we denote its kernel by $\operatorname{ker} M:=\left\{x \in \mathbb{R}^{l} \mid M x=0\right\}$ and its image by $\operatorname{im} M:=\left\{M x \mid x \in \mathbb{R}^{l}\right\}$. Finally, for two linear subspaces $\mathcal{V}_{1}$ and $\mathcal{V}_{2}$ we write $\mathcal{V}_{1} \oplus \mathcal{V}_{2}=\mathcal{V}$, if $\mathcal{V}=\mathcal{V}_{1}+\mathcal{V}_{2}=\left\{v_{1}+v_{2} \mid v_{1} \in \mathcal{V}_{1}, v_{2} \in \mathcal{V}_{2}\right\}$ and $\mathcal{V}_{1} \cap \mathcal{V}_{2}=\{0\}$

\subsection{Linear complementarity problem}

The linear complementarity problem $\operatorname{LCP}(q, M)$ (see [13] for a survey) with data $q \in \mathbb{R}^{k}$ and $M \in \mathbb{R}^{k \times k}$ is the problem of finding $w \in \mathbb{R}^{k}$ such that $0 \leq w \quad q+M w \geq 0$. The solution set of $\operatorname{LCP}(q, M)$ will be denoted by $\operatorname{SOL}(q, M)$. The notation $K(M)$ will denote the set $\{q \mid \operatorname{LCP}(q, M)$ is solvable $\}$.

Let a matrix $M$ of size $k \times k$ and two subsets $I$ and $J$ of $\bar{k}$ of the same cardinality be given. The $(I, J)$ submatrix of $M$ is the submatrix $M_{I J}:=\left(M_{i j}\right)_{i \in I, j \in J}$. The $(I, J)$-minor is defined as the determinant of $M_{I J}$. The $(I, I)$-submatrices and $(I, I)$-minors are also known as the principal submatrices and the principal minors. $M$ is called a nondegenerate matrix if all its principal minors are non-zero. $M$ is called a P-matrix if all its principal minors are positive. P-matrices play an important role in linear complementarity problems, as indicated by the following well known result (cf. [13, thm. 3.3.7]).

Theorem 2.1. For a given matrix $M \in \mathbb{R}^{k \times k}$, the problem $\operatorname{LCP}(q, M)$ has a unique solution for all vectors $q \in \mathbb{R}^{k}$ if and only if $M$ is a P-matrix.

In addition the solutions of $\operatorname{LCP}(q, M)$ depend in a Lipschitz continuous manner on $q$, when $M$ is a P-matrix. To formalize this we define for a given nondegenerate matrix $M \in \mathbb{R}^{m \times m}$

$$
d(M)=\max \left\{\left\|M_{J J}^{-1}\right\| \mid J \subseteq \bar{n}\right\},
$$

where $\|N\|$ denotes the spectral norm $\sqrt{\lambda_{\max }\left(N^{\top} N\right)}$ of a matrix $N$ in which $\lambda_{\max }\left(N^{\top} N\right)$ denotes the largest eigenvalue of $N^{\top} N$.

Lemma 2.2. Assume that $M \in \mathbb{R}^{n \times n}$ is a P-matrix. Let $w^{i}$ be the unique solution of $\operatorname{LCP}\left(q^{i}, M\right)$ for $i=1,2$. Then we have

$$
\left\|w^{1}-w^{2}\right\| \leq d(M)\left\|q^{1}-q^{2}\right\| .
$$

Proof. It is known that the mapping $q \mapsto w$, where $w$ is the unique solution of $\operatorname{LCP}(q, M)$ is a piecewise affine function on a finite number of convex polyhedral cones $P_{J}$ for $J \subseteq \bar{n}$ as given 
in [13, Prop. 1.4.6]. Besides, $q \in P_{J}$ implies that the solution $w$ of the $\operatorname{LCP}(q, M)$ is of the form $w_{J}=-M_{J J}^{-1} q_{j}$ and $w_{\bar{n} \backslash J}=0$. Since the line segment $\left[q^{1}, q^{2}\right]$ lies in only a finite number of these cones (at most $2^{n}$ ), we get the desired inequality.

\subsection{Passivity of a linear system}

We recall the notion of passivity as it is defined in [51] for a linear system $\Sigma(A, B, C, D)$ given by

$$
\dot{x}(t)=A x(t)+B w(t) ; \quad z(t)=C x(t)+D w(t) .
$$

Definition 2.3. [51] The system $\Sigma(A, B, C, D)$ given by (8) is said to be passive if there exists a function $V: \mathbb{R}^{n} \rightarrow \mathbb{R}_{+}$(called a storage function), such that

$$
V\left(x\left(t_{0}\right)\right)+\int_{t_{0}}^{t_{1}} w^{\top}(t) z(t) d t \geq V\left(x\left(t_{1}\right)\right)
$$

holds for all $t_{1} \geq t_{0}$ and for all solutions ${ }^{\dagger}(w, x, z) \in \mathcal{L}_{2}^{m+n+m}\left(t_{0}, t_{1}\right)$ of (8).

The inequality (9) is sometimes called the dissipation inequality. Next, we quote a very well-known characterization of passivity.

Theorem 2.4. [51] The following statements are equivalent.

1. $\Sigma(A, B, C, D)$ is passive.

2. The following matrix inequalities admit a solution $P$ :

$$
P=P^{\top} \geq 0 \text { and }\left(\begin{array}{cc}
A^{\top} P+P A & P B-C^{\top} \\
B^{\top} P-C & -\left(D+D^{\top}\right)
\end{array}\right) \leq 0 .
$$

Moreover, $V(x)=\frac{1}{2} x^{\top} P x$ defines a quadratic storage function if and only if $P$ satisfies the linear matrix inequalities (10).

In case $(A, B, C)$ is minimal and $\Sigma(A, B, C, D)$ is passive, one can actually show that there exists a positive definite solution $P$ to (10), see [51]. We call the latter property passivity with a positive definite storage function, and we will use this frequently in the sequel (as it is weaker than minimality and passivity together). Next we recall the notion of strict passivity of (8), which is slightly stronger than passivity with a positive definite storage function.

Definition 2.5. $\Sigma(A, B, C, D)$ is called strictly passive if the linear matrix inequalities

$$
P=P^{\top}>0 \text { and }\left(\begin{array}{cc}
A^{\top} P+P A+\varepsilon P & P B-C^{\top} \\
B^{\top} P-C & -\left(D+D^{\top}\right)
\end{array}\right) \leq 0 .
$$

have a solution $P$ for some $\varepsilon>0$.

The following technical assumption, which removes specific kinds of redundancy from the system description, will be used below.

\footnotetext{
†Observe that $x$ is absolutely continuous on $\left[t_{0}, t_{1}\right]$ due to $w \in \mathcal{L}_{2}^{m}\left(t_{0}, t_{1}\right)$.
} 
Assumption 2.6. $\left(\begin{array}{c}B \\ D+D^{\top}\end{array}\right)$ has full column rank.

Passivity with a positive definite storage function of a system has some useful implications for the subsystems, as formulated below.

Lemma 2.7. Consider a matrix quadruple $(A, B, C, D)$ satisfying Assumption 2.6. If the system $\Sigma(A, B, C, D)$ is passive with a positive definite storage function, then the following statements hold for each $J \subseteq \bar{m}$, where $P$ is a positive definite solution to the LMIs (10).

1. The matrix $D_{J J}$ is nonnegative definite.

2. If $w^{\top} D_{J J} w=0$ then $P B_{\bullet} w=\left(C_{J}\right)^{\top} w$.

3. If $w^{\top} D_{J J} w=0$ and $w \neq 0$, then $w^{\top} C_{J \bullet} B_{\bullet} w>0$.

4. The rational matrix function $s^{-1}\left(D_{J J}+C_{J \bullet} B_{\bullet} s^{-1}\right)^{-1}$ is proper.

5. The matrix $D_{J J}+C_{J \bullet} B_{\bullet} \sigma^{-1}$ is positive definite for all sufficiently large $\sigma \in \mathbb{R}$.

The proof of this result was given in [11] under Assumption 2.6 and the assumption of minimality and passivity of $(A, B, C, D)$. However, the proof in [11] essentially only uses Assumption 2.6 and passivity with a positive definite storage function, which establishes Lemma 2.7.

3. Well-posedness for index-one LCS with inputs

\subsection{Initial solutions}

We use a solution concept for the LCS (1) that is built on the notion of an "initial solution". This term refers to trajectories that satisfy the dynamics of one mode (2) only and that satisfy the inequality conditions in (1) possibly only on an interval of finite length. We employ the theory of distributions in formalizing the solution concept, since the abrupt changes in the trajectories (e.g. impacts in mechanics) can be modeled adequately by Dirac impulses (see also Example 3.4 below). We now recall the concepts of Bohl distribution and initial solution [25]. We use typewriter font when we want to emphasize the distributional nature of trajectories. Recall that $\mathcal{B}$ denotes the set of Bohl functions (i.e. functions having strictly proper rational Laplace transforms) defined on $\mathbb{R}_{+}$such as exponentials, sines, cosines, polynomials and their sums and products.

Definition 3.1. A distribution $\mathrm{w}$ is said to be a Bohl distribution if $\mathrm{w}=\mathrm{w}_{\text {imp }}+\mathrm{w}_{\text {reg }}$ with $\mathrm{w}_{\text {imp }}=$ $\sum_{i=0}^{l} w^{-i} \delta_{0}^{(i)}$ for $w^{-i} \in \mathbb{R}$ and $\mathrm{w}_{\text {reg }} \in \mathcal{B}$. We call $\mathrm{w}_{\text {imp }}$ the impulsive part of $\mathrm{w}$ and $\mathrm{w}_{\text {reg }}$ the regular part of $\mathrm{w}$. The space of all Bohl distributions is denoted by $\mathcal{B}_{i m p}$.

Note that Bohl distributions have rational Laplace transforms. One may call a (smooth) Bohl function $w \in \mathcal{B}$ initially nonnegative if there exists an $\varepsilon>0$ such that $w(t) \geq 0$ for all $t \in[0, \varepsilon)$. Note that a Bohl function $w$ is initially nonnegative if and only if there exists a $\sigma_{0} \in \mathbb{R}$ such that its Laplace transform satisfies $\hat{w}(\sigma) \geq 0$ for all $\sigma \geq \sigma_{0}$. Hence, there is a connection between small time values for time functions and large values for the indeterminate $s$ in the Laplace transform. This fact is closely related to the well-known initial value theorem. The definition of initial nonnegativity for Bohl distributions will be based on this observation (see also [25]).

Definition 3.2. We call a Bohl distribution w initially nonnegative, if its Laplace transform $\hat{w}(s)$ satisfies $\hat{w}(\sigma) \geq 0$ for all sufficiently large real $\sigma$. 
To relate the definition to the time domain, note that a scalar-valued Bohl distribution w without derivatives of the Dirac impulse (i.e. $\mathrm{w}_{i m p}=w^{0} \delta$ for some $w^{0} \in \mathbb{R}$ ) is initially nonnegative if and only if either $w^{0}>0$, or $w^{0}=0$ and there exists an $\varepsilon>0$ such that $\mathrm{w}_{\text {reg }}(t) \geq 0$ for all $t \in[0, \varepsilon)$. With these notions we can recall the concept of an initial solution [25]. Loosely speaking, an initial solution to (1) with initial state $x_{0}$ and Bohl input $u \in \mathcal{B}^{k}$ is a triple (w, x, z) $\in \mathcal{B}_{i m p}^{m+n+m}$ that satisfies (2) for some mode $I$ and that satisfies (3) either during a time interval of positive length or at a time instant where a Dirac distribution is active (as formalized in the notion of initial nonnegativity).

We now proceed to define the notion of initial solutions for LCS. Later we define full solutions to LCS as concatenations of time-shifted initial conditions; see Def. 3.16 below. We use Bohl functions as inputs, which allows us to prove that solutions can be found in the class Bohl distributions. Later on, in the global solution concept, we admit inputs that are themselves concatenations of Bohl functions. This input class is sufficient in an environment in which signals are generated by linear systems, and it allows us to use algebraic tools which involve rational functions so that we can stay within a framework that is familiar to linear system theorists.

Definition 3.3. A distribution ( $\mathrm{w}, \mathrm{x}, \mathrm{z}) \in \mathcal{B}_{i m p}^{m+n+m}$ is said to be an initial solution to (1) with initial state $x_{0}$ and input $u \in \mathcal{B}^{k}$ if

1. $\dot{\mathrm{x}}=A \mathrm{x}+B \mathrm{w}+E u+x_{0} \delta_{0}$ and $\mathrm{z}=C \mathrm{x}+D \mathrm{w}+F u$ as equalities of distributions.

2. there exists an $J \subseteq \bar{m}$ such that $\mathrm{w}_{i}=0, i \in I^{c}$ and $\mathrm{z}_{i}=0, i \in J$ as equalities of distributions.

3. $w$ and $z$ are initially nonnegative.

The statements 1 and 2 in the definition above express that an initial solution follows the dynamics (2) for mode $J$ on the semi-infinite time interval $\mathbb{R}_{+}$, and satisfies the initial condition $x(0)=x_{0}$.

Example 3.4. Consider the system $\dot{x}(t)=w(t), z(t)=x(t)$ together with (1c). This represents an electrical network consisting of a capacitor connected to a diode. The current in the network is equal to $w$ and the voltage across the capacitor is equal to $z=x$. For initial state $x(0)=1,(\mathrm{w}, \mathbf{x}, \mathbf{z})$ with $\mathrm{w}=0$ and $\mathrm{z}(t)=\mathrm{x}(t)=1$ for all $t \in \mathbb{R}$ is an initial solution. This corresponds to the case in which the diode is blocking and there is no (nonzero) current in the network. To show that the distributional framework is convenient, consider the initial state $x(0)=-1$, for which $(\mathrm{w}, \mathrm{x}, \mathrm{z})$ with $\mathrm{w}=\delta, \mathrm{x}(t)=\mathrm{z}(t)=0$, $t>0$ is the unique initial solution. This corresponds to an instantaneous discharge of the capacitor at time instant 0 resulting in a state jump from $x(0)=-1$ to 0 at time 0 induced by the impulse.

This example indicates that there is a clear physical interpretation of the impulses in initial solutions to model the abrupt changes in trajectories of electrical circuits (see also [11,21,22]). Also for mechanical systems, these initial solutions induce state jumps with a clear physical meaning (related to inelastic restitution laws) as shown in [25].

\subsection{Initial well-posedness and local well-posedness}

In this subsection we derive novel conditions that guarantee existence and uniqueness of so-called initial solutions. These results will be exploited to obtain first a local well-posedness result and subsequently a global one. The notion of "index" of the transfer matrix $C(s I-A)^{-1} B+D$ will play an important role.

Definition 3.5. A rational matrix $H(s) \in \mathbb{R}^{l \times l}(s)$ is said to be of index $r$, if it is invertible as a rational matrix and $s^{-r} H^{-1}(s)$ is proper. It is said to be totally of index $r$, if all its principal submatrices $H_{J J}(s)$ for $J \subset \bar{l}$ are of index $r$. 
Before we derive well-posedness results based on low-index conditions, we first present some characterizations of rational matrices of low index. The index of a rational matrix is characterized in terms of its power series expansion around infinity in the following lemma. The proof of the lemma can be found in the Appendix.

Lemma 3.6. Let $H(s) \in \mathbb{R}^{l \times l}(s)$ be given and let its power series expansion around infinity be given by

$$
H(s)=H^{0}+H^{1} s^{-1}+\cdots .
$$

Then the following statement are equivalent.

1. $H(s)$ is of index 1 .

2. $H^{0}+H^{1} s^{-1}$ is of index 1 .

3. im $H^{0} \oplus H^{1}\left(\operatorname{ker} H^{0}\right)=\mathbb{R}^{l}$.

4. There exist matrices $P \in \mathbb{R}^{p \times l}$ and $Q \in \mathbb{R}^{(l-p) \times l}$ such that

$$
\left[\begin{array}{l}
P \\
Q
\end{array}\right] \text { and }\left[\begin{array}{l}
P H^{0} \\
Q H^{1}
\end{array}\right]
$$

are both nonsingular and $Q H^{0}=0$.

In particular, the constant $d(\cdot)$ of index 1 rational matrices will be of interest.

Lemma 3.7. Let $H(s) \in \mathbb{R}^{l \times l}(s)$ be totally of index 1 . Then there exists an $\alpha>0$ such that $d(H(\sigma)) \leq \alpha \sigma$ for all sufficiently large $\sigma$.

Proof. Note that $H_{J J}(s)$ is of index 1 for each index set $J \subseteq \bar{m}$ by the definition of total index. Hence, $s^{-1} H_{J J}^{-1}(s)$ is proper for each $J \subseteq \bar{m}$. Therefore, for each $J \subseteq \bar{m}$ there exists $\alpha_{J}>0$ such that

$$
\left\|H_{J J}^{-1}(\sigma)\right\| \leq \alpha_{J} \sigma
$$

for all sufficiently large $\sigma$. As a consequence, we have $d(H(\sigma)) \leq \alpha \sigma$ for all sufficiently large $\sigma$ where $\alpha=\max _{J \subseteq \bar{m}} \alpha_{J}$.

Theorem 3.8. Consider an LCS with external inputs given by (1) such that $G(s)=C(s I-A)^{-1} B+D$ is totally of index 1 and $G(\sigma)$ is a P-matrix for sufficiently large $\sigma$. Define $\mathcal{Q}_{D}:=S O L(0, D)=\{v \in$ $\left.\mathbb{R}^{m} \mid 0 \leq v \perp D v \geq 0\right\}$ and let $K(D)$ be the set $\left\{q \in \mathbb{R}^{m} \mid L C P(q, D)\right.$ solvable $\}$.

1. For arbitrary initial state $x_{0} \in \mathbb{R}^{n}$ and any input $u \in \mathcal{B}^{k}$, there exists exactly one initial solution, which will be denoted by $\left(\mathrm{w}^{x_{0}, u}, \mathrm{x}^{x_{0}, u}, \mathbf{z}^{x_{0}, u}\right)$.

2. No initial solution contains derivatives of the Dirac distribution. Moreover,

$$
\mathrm{w}_{i m p}^{x_{0}, u}=w^{0} \delta_{0} ; \mathbf{x}_{i m p}^{x_{0}, u}=0 ; \mathbf{z}_{i m p}^{x_{0}, u}=D w^{0} \delta_{0} \text { for some } w^{0} \in \mathcal{Q}_{D} .
$$

3. For all $x_{0} \in \mathbb{R}^{n}$ and $u \in \mathcal{B}^{k}$, we have $C x_{0}+F u(0)+C B w^{0} \in K(D)$.

4. The initial solution $\left(\mathrm{w}^{x_{0}, u}, \mathrm{x}^{x_{0}, u}, \mathbf{z}^{x_{0}, u}\right)$ is smooth (i.e., has a zero impulsive part) if and only if $C x_{0}+F u(0) \in K(D)$. 
Based on this result we can conclude that the impulsive part of $\mathrm{w}_{i m}$ is given by $\mathrm{w}_{i m p}^{x_{0}, u}=w^{0} \delta_{0}$. From Def. 3.3 it follows that this impulsive part induces a state jump from $x_{0}$ to $x_{0}+B w^{0}$, which in circuits might correspond to infinitely large currents related to instantaneous discharges of capacitors (see Example 3.4) and in mechanical systems to infinite reaction forces that cause resets in the velocities of impacting bodies.

To prove this theorem, we need to recall the so-called rational complementarity problem [24] and its relation to the initial solution.

Problem 3.9. $\left(\operatorname{RCP}\left(x_{0}, \hat{u}(s), A, B, C, D, E, F\right)\right)$ Given $\quad x_{0} \quad \in \quad \mathbb{R}^{n}, \hat{u}(s) \in \mathbb{R}^{p}(s)$, and $(A, B, C, D, E, F)$ with $A \in \mathbb{R}^{n \times n}, B \in \mathbb{R}^{n \times m}, C \in \mathbb{R}^{m \times n}, D \in \mathbb{R}^{m \times m}, E \in \mathbb{R}^{n \times p}$ and $F \in \mathbb{R}^{m \times p}$ find $\hat{w}(s) \in \mathbb{R}^{m}(s)$ such that

1. $\hat{w}(s) \perp \hat{z}(s)$ for all $s \in \mathbb{C}$.

2. $\hat{w}(\sigma) \geq 0$ and $\hat{z}(\sigma) \geq 0$ for all sufficiently large $\sigma \in \mathbb{R}$

where

$$
\hat{z}(s)=C(s I-A)^{-1} x_{0}+\left[D+C(s I-A)^{-1} B\right] \hat{w}(s)+\left[F+C(s I-A)^{-1} E\right] \hat{u}(s) .
$$

For brevity of notation, we denote $\operatorname{RCP}\left(x_{0}, \hat{u}(s), A, B, C, D, E, F\right)$ by $\operatorname{RCP}\left(x_{0}, \hat{u}(s)\right)$ if the sixtuple $(A, B, C, D, E, F)$ is clear from the context. There is one-to-one correspondence between the solutions of RCP and initial solutions of LCS as described in the following proposition.

Proposition 3.10. Consider a matrix sixtuple $(A, B, C, D, E, F)$. The following statements hold.

1. Let $\hat{w}(s)$ be a solution of $\operatorname{RCP}\left(x_{0}, \hat{u}(s)\right)$ for some $x_{0}$ and strictly proper $\hat{u}(s)$. Define $\hat{x}(s)$ and $\hat{z}(s)$ as follows:

$$
\begin{aligned}
& \hat{x}(s)=(s I-A)^{-1} x_{0}+(s I-A)^{-1} B \hat{w}(s)+(s I-A)^{-1} E \hat{u}(s) \\
& \hat{z}(s)=C \hat{x}(s)+D \hat{w}(s)+F \hat{u}(s) .
\end{aligned}
$$

Then, the inverse Laplace transform $(\mathrm{w}, \mathrm{x}, \mathbf{z})$ of $(\hat{w}(s), \hat{x}(s), \hat{z}(s))$ is an initial solution with the initial state $x_{0}$ and input $u$ where $u$ is the inverse Laplace transform of $\hat{u}(s)$.

2. Let $(\mathrm{w}, \mathrm{x}, \mathrm{z})$ be an initial solution with the initial state $x_{0}$ and input $u$. Also let $\hat{w}(s)$ be the Laplace transform of $\mathrm{w}$. Then, $\hat{w}(s)$ solves $\operatorname{RCP}\left(x_{0}, \hat{u}(s)\right)$, where $\hat{u}(s)$ is the Laplace transform of $u$.

Proof. Evident from the proof of [25, Thm. 5.3].

In the proof of Thm.3.8, we also need the following two lemmas whose proofs are given in the Appendix.

Lemma 3.11. Consider an LCS with external inputs given by (1). Suppose that for the initial state $x_{0} \in \mathbb{R}^{n}$ and the input $u \in \mathcal{B}$ there exists an initial solution $(\mathrm{w}, \mathbf{x}, \mathbf{z})$ with $\mathrm{w}_{i m p}=w^{0} \delta_{0}$. Then the following properties hold.

(i) $w^{0} \in \mathcal{Q}_{D}, \mathbf{x}_{i m p}=0$ and $\mathbf{z}_{i m p}=D w^{0} \delta_{0}$.

(ii) $C x_{0}+F u(0)+C B w^{0} \in K(D)$.

(iii) If $\left(D+D^{\top}\right) v=0$ for all $v \in \mathcal{Q}_{D}$, then $w^{0} \perp C x_{0}+F u(0)+C B w^{0}$.

Lemma 3.12. Consider an LCS with external inputs given by (1) such that $G(s)=C(s I-A)^{-1} B+D$ is totally of index 1 and $G(\sigma)$ is a P-matrix for sufficiently large $\sigma$. Then the following statements hold. 
(i) $\operatorname{RCP}\left(x_{0}, \hat{u}(s)\right)$ has a unique solution for all $x_{0} \in \mathbb{R}^{n}$ and for all $\hat{u}(s) \in \mathbb{R}^{p}(s)$.

(ii) For a given strictly proper $\hat{u}(s)$, the unique solution of $\operatorname{RCP}\left(x_{0}, \hat{u}(s)\right)$ is strictly proper if and only if $C x_{0}+F u(0) \in K(D)$, where $u$ is the inverse Laplace transform of $\hat{u}(s)$.

We can now prove Thm. 3.8.

Proof of Theorem 3.8.

1: This follows from Lemma 3.12 item (i) and Proposition 3.10.

2: Let $(\hat{w}(s), \hat{z}(s))$ be the Laplace transform of $\left(\mathrm{w}^{x_{0}, w}, \mathrm{z}^{x_{0}, w}\right)$. Proposition 3.10 item 2 and the formulation of the RCP imply that there exists an index set $J \subseteq \bar{m}$ such that

$$
\begin{gathered}
\hat{w}_{\bar{m} \backslash J}(s) \equiv 0 \\
\hat{z}_{J}(s)=C_{J \bullet}(s I-A)^{-1} x_{0}+H_{J}(s) \hat{u}(s)+G_{J J}(s) \hat{w}_{J}(s) \equiv 0,
\end{gathered}
$$

where $G(s)=D+C(s I-A)^{-1} B$ and $H(s)=F+C(s I-A)^{-1} E$. By solving (15) for $\hat{u}_{J}(s)$, we get

$$
\hat{w}_{J}(s)=-G_{J J}^{-1}(s)\left[C_{J}(s I-A)^{-1} x_{0}+H_{J} \bullet(s) \hat{u}(s)\right] .
$$

Note that the second factor of the above equality is strictly proper. Since $s^{-1} G_{J J}^{-1}(s)$ as $G(s)$ is totally of index $1, \hat{w}(s)$ must be proper. Therefore, its inverse Laplace transform $\mathrm{w}^{x_{0}, w}$ cannot contain derivatives of the Dirac distribution. Let $\mathrm{w}_{i m p}^{x_{0}, w}=w^{0} \delta$ for some $w^{0} \in \mathbb{R}^{m}$. Using now Lemma 3.11 completes the proof of this statement.

3: This follows from Lemma 3.11.

4: This follows from Lemma 3.12 item (ii) and Proposition 3.10.

Theorem 3.8 gives explicit conditions for existence and uniqueness of solutions. The second statement indicates that derivatives of Dirac distributions are absent in the behavior of LCS for which $G(s)=$ $C(s I-A)^{-1} B+D$ is totally of index 1 . The fourth statement gives necessary and sufficient condition for an initial solution to be smooth. In particular, a LCS satisfying the conditions of Theorem 3.14 is "impulse-free" (no state jumps), if $\mathcal{Q}_{D}=\operatorname{SOL}(0, D)=\{0\}$ (or, in terms of [13], if $D$ is an $\mathrm{R}_{0}$-matrix) or $K(D)=\mathbb{R}^{m}$ (in terms of [13] this means that the matrix $D$ is a so-called $Q$ matrix). In case the matrix $\left[\begin{array}{ll}C & F]\end{array}\right.$ has full row rank, this condition is also necessary. Other sufficient conditions, that are easier to verify, are $D$ being a positive definite matrix, $D$ nonnegative definite and $\operatorname{Ker}\left(D+D^{\top}\right) \cap \mathbb{R}_{+}^{m}=\{0\}$ or $w^{\top} D w>0$ for all $w \in \mathbb{R}_{+}^{m}$ and $w \neq 0$.

The first statement in Theorem 3.8 by itself does not immediately guarantee the existence of a solution on a time interval of positive length. The reason is that an initial solution with a non-zero impulsive part may only be valid at the time instant on which the Dirac distribution is active. If the impulsive part of the (unique) initial solution is equal to $w^{0} \delta_{0}$, the state after re-initialization is equal to $x_{0}+B w^{0}$. From this "next" initial state again an initial solution has to be determined, which might in principle also have a non-zero impulsive part, which results in another state jump. As a consequence, the occurrence of infinitely many jumps at $t=0$ without any smooth continuation on a positive length time interval is in principle possible (sometimes called "livelock" in hybrid systems theory, which is a 
particular case of Zeno behavior ${ }^{\ddagger}$ ). However, Theorem 3.8 excludes this kind of Zeno phenomenon: if smooth continuation is not directly possible from $x_{0}$, it is possible after one re-initialization. Indeed, since $C\left(x_{0}+B w^{0}\right)+F u(0)=C x_{0}+F u(0)+C B w^{0} \in K(D)$, it follows from the fourth claim that the initial solution corresponding to $x_{0}+B w^{0}$ and input $u$ is smooth. This initial solution satisfies the (in)equalities in (1) on an interval of the form $(0, \varepsilon)$ with $\varepsilon>0$ by definition and hence, we proved a local existence and uniqueness result. However, we still have to show global existence of solutions as other kinds of Zeno behavior (accumulation of mode switching times without continuation beyond the right accumulation time) might prevent this.

Remark 3.13. Theorem 3.8 extends earlier results in $[11,21,24]$ that were based on passivity, as formulated below. The proof of Theorem 3.14 (cf. [11,21]) can be based on Lemma 2.7; for brevity we do not demonstrate this here.

Theorem 3.14. Consider an LCS such that $(A, B, C, D)$ is passive with a positive definite storage function and Assumption 2.6 holds. Then $G(s)=C(s I-A)^{-1} B+D$ is totally of index 1 , and both $G(\sigma)$ and $D+s^{-1} C B$ are P-matrices for sufficiently large $\sigma$. In this case the results of Theorem 3.8 hold for (1) with $K(D)=\mathcal{Q}_{D}^{*}$.

\subsection{Global well-posedness}

Before we can formulate a global well-posedness theorem, we need to define a class of allowable input functions and the global solution concept.

Definition 3.15. A function $u: \mathbb{R}_{+} \rightarrow \mathbb{R}$ is called a piecewise Bohl function, if

1. for all $\tau \in \mathbb{R}_{+}$there are $\varepsilon>0$ and $v \in \mathcal{B}$ such that $u(t)=v(t)$ for all $t \in[\tau, \tau+\varepsilon)$,

2. $u$ is locally bounded in the sense that for any $T \in \mathbb{R}_{+}$there is a constant $M$, possibly depending on $T$, such that $|u(t)| \leq M$ for all $t \in[0, T]$, and

3. the set of discontinuity points of $u$ is isolated ${ }^{\S}$.

The set of all piecewise Bohl functions is denoted by $\mathcal{P B}$. A distribution $u: \mathbb{R}_{+} \rightarrow \mathbb{R}$ is called piecewise Bohl with first order impulses or just simply piecewise Bohl if $\mathrm{u}$ is the sum of a piecewise Bohl function $\mathrm{u}_{r e g}$ and the distribution $\mathrm{u}_{i m p}=\sum_{\theta \in \Gamma} w^{\theta} \delta_{\theta}$, where $\Gamma=\left\{\tau_{i}\right\}_{i}$ is an isolated subset of $\mathbb{R}_{+}$. This distribution space will be denoted by $\mathcal{P} \mathcal{B}_{0}$.

Definition 3.16. Let $(\mathrm{w}, \mathrm{x}, \mathrm{z}) \in \mathcal{P B}_{0}^{m+n+m}$ be given with

$$
\mathrm{w}_{i m p}=\sum_{\theta \in \Gamma} w^{\theta} \delta_{\theta}, \mathbf{x}_{i m p}=\sum_{\theta \in \Gamma} x^{\theta} \delta_{\theta}, \mathbf{z}_{i m p}=\sum_{\theta \in \Gamma} z^{\theta} \delta_{\theta}
$$

for $w^{\theta} \in \mathbb{R}^{m}, x^{\theta} \in \mathbb{R}^{n}$ and $w^{\theta} \in \mathbb{R}^{m}$ for $\theta \in \Gamma$, where $\Gamma$ is an isolated subset of $\mathbb{R}_{+}$. Then we call (w, x, z) a (global) solution to LCS (1) with input function $u \in \mathcal{P B}$ and initial state $x_{0}$, if the following properties hold.

\footnotetext{
$\ddagger$ Zeno behavior means that the system under study has solutions that have an infinite number of discrete events (such as state jumps) within a time interval of finite length.

${ }^{\S} \mathrm{A}$ set $\Gamma \subseteq \mathbb{R}$ is said to be isolated if for all $\tau \in \Gamma$ there is an $\varepsilon>0$ such that $\Gamma \cap(\tau-\varepsilon, \tau+\varepsilon)=\{\tau\}$. 
1. For any interval $(a, b)$ such that $(a, b) \cap \Gamma=\emptyset$ the restriction $\left.\mathbf{x}_{r e g}\right|_{(a, b)}$ is absolutely continuous and satisfies (1) for almost all $t \in(a, b)$.

2. For each $\theta \in \Gamma$ the corresponding impulse $\left(w^{\theta} \delta_{\theta}, x^{\theta} \delta_{\theta}, z^{\theta} \delta_{\theta}\right)$ is equal to the impulsive part of an initial solution ${ }^{\top}$ to (1) with initial state $\mathrm{x}_{r e g}(\theta-):=\lim _{t \uparrow \theta} \mathrm{x}_{r e g}(t)$ (taken equal to $x_{0}$ for $\theta=0$ ) and input $t \mapsto u(t-\theta)$.

3. For times $\theta \in \Gamma$ the equality $\mathrm{x}_{r e g}(\theta+)=\mathrm{x}_{r e g}(\theta-)+B w^{\theta}$ holds where $w^{\theta}$ the multiplier of Dirac pulse supported at $\theta$.

Theorem 3.17. Consider an LCS with external inputs given by (1) such that $G(s)=C(s I-A)^{-1} B+$ $D$ is totally of index 1 and $G(\sigma)$ is a P-matrix for sufficiently large $\sigma$. The LCS (1) has a unique (global) solution $(\mathrm{w}, \mathrm{x}, \mathrm{z}) \in \mathcal{P} \mathcal{B}_{0}^{k+n+k}$ for any initial state $x_{0}$ and input $u \in \mathcal{P} \mathcal{B}^{k}$. Moreover, $\mathrm{x}_{i m p}=0$ and impulses in $(\mathrm{w}, \mathrm{z})$ can only show up at the initial time and times for which $F u$ is discontinuous.

Proof. The proof is based on the local existence result (Theorem 3.8) and the construction of global solutions by concatenation of initial solutions along the lines of the proof as in the passive case [11, Thm. 7.5] by incorporating the presence of impulses; we do not repeat the reasoning here in the interest of brevity.

This theorem implies that if $F u$ is continuous, jumps of the state can only occur at the initial time instant 0 .

Remark 3.18. In this paper we focus on the class of piecewise Bohl functions and piecewise Bohl distributions (with first order impulses) as in Definition 3.15 as inputs and solution trajectories, respectively. Although more general solution classes are available such as, for instance, measuredriven solutions (see, e.g., $[1,10,31,34,35,46]$ ) that are able to encompass richer behavior such as leftaccumulations in the mode-switching times, the more restricted class of piecewise Bohl distributions is natural in the context of PWA systems and LCS and clearly suits our purposes. In particular, the properties of $\mathcal{P} \mathcal{B}_{0}$ fit the hybrid perspective on the construction of trajectories (time intervals of smooth evolution separated by discrete events such as jumps and mode switches) and lead to compact and algebraic well-posedness conditions in the end. The existence result of Theorem 3.17 indicates that we can think of an LCS (for given initial conditions) as a mapping from piecewise Bohl distributions to piecewise Bohl distributions, just as we can think of classical linear systems as mappings from Bohl functions to Bohl functions. Given that we have an existence result in terms of piecewise Bohl solutions, any uniqueness result in a wider space will produce a proof that solutions in this wider space must in fact be piecewise Bohl. A result of this type has been provided in [21], where it has been shown under a passivity assumption solutions to LCS are unique within an extension class constructed from $\mathcal{L}_{2}$-functions. For uniqueness results see also [46]. An interesting question, which we do not answer in this paper, is whether uniqueness within (say) the extension class based on $\mathcal{L}_{2}$-functions is still valid if passivity is replaced by the assumptions that we impose in this paper on the closed-loop system.

9 Note that we shift time by $\theta$ to be able to use the definition of an initial solution, which is given for an initial condition at $t=0$. 


\section{Observer design}

Consider the LCS (1) and assume that there is no direct observation of the state variable; instead, only an output variable given by

$$
y(t)=G x(t) \in \mathbb{R}^{p}
$$

is measured. Based on this observed output, we aim at estimating the continuous state $x(t)$ of (1) using an observer. For the observer design to be meaningful, we have to assume some conditions of the existence of solutions to the observed system (1).

Assumption 4.1. For any initial state $x_{0}$ and for any input function $u \in \mathcal{P} \mathcal{B}^{k}$ there exists a global solution $(\mathrm{w}, \mathrm{x}, \mathrm{z}) \in \mathcal{P} \mathcal{B}_{0}^{m+n+m}$ to system (1) in the sense of Definition 3.16.

The well-posedness theory derived before can be used to guarantee this property. For instance, Theorem 3.17 shows that under the assumption that $G(s)=C(s I-A)^{-1} B+D$ is totally of index 1 and $G(\sigma)$ is a P-matrix for sufficiently large $\sigma$, Assumption 4.1 is indeed satisfied.

We propose the following observer for the LCS (1) with measured output (16):

$$
\begin{aligned}
& \dot{\hat{x}}(t)=A \hat{x}(t)+B \hat{w}(t)+E u(t)+L(y(t)-\hat{y}(t)) \\
& \hat{z}(t)=C \hat{x}(t)+D \hat{w}(t)+F u(t) \\
& 0 \leq \hat{z}(t) \perp \hat{w}(t) \geq 0 \\
& \hat{y}(t)=G \hat{x}(t)
\end{aligned}
$$

with the observer gain $L$. The design condition that we will use is stated as follows.

Condition 4.2. The matrix $L$ is chosen such that the four-tuple $(A-L G, B, C, D)$ is strictly passive.

First of all, we show that the observer structure (17) produces values $\hat{x}$, i.e. existence of global solutions to (17) is guaranteed given an initial estimate $\hat{x}_{0}$ and external inputs $u \in \mathcal{P} \mathcal{B}^{k}$ and $y \in \mathcal{P} \mathcal{B}^{p}$. Using Theorem 3.14 and Theorem 3.17 we obtain the following result.

Theorem 4.3. Consider the observer (17) with external inputs $u \in \mathcal{P B}^{k}$ and $y \in \mathcal{P B}^{p}$, where $y$ is obtained from the LCS given by (1) and (16) for some initial state $x_{0}$ and input $u$. If Assumption 2.6 and Condition 4.2 are satisfied, then for any initial state $\hat{x}_{0}$ there exists a unique global solution $(\hat{\mathrm{w}}, \hat{\mathrm{x}}, \hat{\mathrm{z}})$ to (17).

Since we proved global existence of $\hat{x}$, we can consider the observation error $e:=x-\hat{x}$, which evolves according to the following dynamics:

$$
\begin{aligned}
& \dot{e}(t)=(A-L G) e(t)+B w(t)-B \hat{w}(t) \\
& z(t)=C x(t)+D w(t)+F u(t) \\
& \hat{z}(t)=C \hat{x}(t)+D \hat{w}(t)+F u(t) \\
& 0 \leq z(t) \perp w(t) \geq 0, \text { and } 0 \leq \hat{z}(t) \perp \hat{w}(t) \geq 0 .
\end{aligned}
$$

We apply the following notion of stability, which only calls for convergence of certain output variables, but which on the other hand leaves freedom in the choice of input functions. While such a definition may be formulated in a general context of input/output dynamical systems, we state a specific form here so that we can more easily accommodate the distributional spaces that we work with. 
Definition 4.4. The LCS (1) is called globally exponentially stable (GES) in the variable $e=H x$ for some $H \in \mathbb{R}^{q \times n}$, if the following conditions hold.

Global existence: For each $x_{0}$ and input $u \in \mathcal{P} \mathcal{B}^{k}$ there exists a global solution to (1) in the sense of Definition 3.16.

Exponential decay : There are positive constants $c$ and $\mu$ such that for any initial state $x_{0}$ and input $u \in \mathcal{P} \mathcal{B}^{k}$ all corresponding global solutions $\left(\mathrm{w}, \mathrm{x}, \mathrm{z}\right.$ ) satisfy $\left\|\mathrm{e}_{\text {reg }}(t)\right\| \leq c e^{-\mu t}\left\|e_{0}\right\|$ for all $t \geq 0$, where $e_{0}=H x_{0}$ and $\mathrm{e}_{r e g}=H \mathbf{x}_{r e g}$.

The LCS (1) is called globally exponentially stable (GES) if it is GES in $e=x(H=I)$.

The definition of GES is formulated for systems with inputs, since the error dynamics is given by (18) and (1) (or (17)), and therefore not autonomous. For the case of linear systems the error dynamics is autonomous, but for nonlinear and hybrid systems it is not. For piecewise linear systems this was pointed out also in $[29,38,47]$ together with discussions on the complexity it causes for guaranteeing stability of the error dynamics. In addition, the GES of the estimation error dynamics requires a kind of "partial stability" in the sense that although $e$ and $x$ (or $\hat{x}$ ) are the state variables in (18) and (1) (or (17)), the aim is only to prove stability with respect to $e$. Therefore, we defined GES for certain variables in the system. Note that in case of autonomous systems the above definition of GES reduces to the standard one.

Theorem 4.5. Consider the error dynamics (18) with (1) (or (17)) for input $u \in \mathcal{P B}^{k}$ such that Assumption 2.6, Assumption 4.1 and Condition 4.2 hold. Then the error dynamics is GES in $e$.

Proof. Consider the Lyapunov function $V_{e}(e)=e^{\top} P e$ with $P$ satisfying

$$
P=P^{\top}>0 \text { and }\left(\begin{array}{cc}
(A-L G)^{\top} P+P(A-L G)+\varepsilon P & P B-C^{\top} \\
B^{\top} P-C & -\left(D+D^{\top}\right)
\end{array}\right) \leq 0
$$

for some $\varepsilon>0$. Then we can compute the (right) derivative of $V_{e}$ at the continuity points of the trajectory $e$ satisfying (18). This yields

$$
\begin{aligned}
\dot{V}_{e}(e)= & e^{\top}(A-L G)^{\top} P e+(w-\hat{w})^{\top} B^{\top} P e+e^{\top} P(A-L G) e+e^{\top} P B(w-\hat{w}) \\
= & \left(\begin{array}{c}
e \\
w-\hat{w}
\end{array}\right)^{\top}\left(\begin{array}{cc}
(A-L G)^{\top} P+P(A-L G) & P B-C^{\top} \\
B^{\top} P-C & -\left(D+D^{\top}\right)
\end{array}\right)\left(\begin{array}{c}
e \\
w-\hat{w}
\end{array}\right)+ \\
& +2(w-\hat{w})^{\top}[C(x-\hat{x})+D(w-\hat{w})] .
\end{aligned}
$$

Since $z-\hat{z}=C(x-\hat{x})+D(w-\hat{w})$, it follows from the complementarity conditions (18d) that $(w-\hat{w})^{\top}[C(x-\hat{x})+D(w-\hat{w})] \leq 0$. Hence, from (19), it now follows that $\dot{V}_{e}(e(t)) \leq-\varepsilon V_{e}(e(t))$ at times $t$ where no jump occurs.

As derived in Theorem 3.17 jumps occur in the observed system (1) and in the observer dynamics (17) at the initial time 0 or at discontinuity points of $F u$ only. Hence, we only have to consider jumps in $e$ for the initial time 0 and discontinuity points of $F u$. We consider first the initial time 0 and consider an initial solution from the states $x_{0}$ and $\hat{x}_{0}$, respectively. From Theorem 3.8 it is clear a state jump occurs from $x_{0}$ to $x_{0}+B w^{0}$, where $w^{0} \delta$ is the impulsive part of the initial solution to (1) with input $u$, and a state jump occurs from $\hat{x}_{0}$ to $\hat{x}_{0}+B \hat{w}^{0}$, where $\hat{w}^{0} \delta$ is the impulsive part of the initial solution to (17) with input $u$ and $y$. Hence, the value of $e(0-)$ (before the jump) is equal to $x_{0}-\hat{x}_{0}$ and after 
the jump it is equal to $x_{0}-\hat{x}_{0}+B\left(w^{0}-\hat{w}^{0}\right)$. This means that

$$
\begin{aligned}
V_{e}(e(0+))-V_{e}(e(0-))= & \left(x_{0}-\hat{x}_{0}\right)^{\top} P B\left(w^{0}-\hat{w}^{0}\right)+ \\
& +\left(w^{0}-\hat{w}^{0}\right)^{\top} B^{\top} P\left(x_{0}-\hat{x}_{0}\right)+\left(w^{0}-\hat{w}^{0}\right)^{\top} B^{\top} P B\left(w^{0}-\hat{w}^{0}\right) .
\end{aligned}
$$

Since (19) holds it is clear that $D+D^{\top}$ is a nonnegative definite matrix. Since Theorem 3.8 and Lemma 3.11 show that $w^{0}$ and $\hat{w}^{0}$ are both in $\mathcal{Q}_{D}$, it follows that $(D+D)^{\top} w^{0}=(D+D)^{\top} \hat{w}^{0}=0$. This implies via (19) that $C^{\top} w^{0}=P B w^{0}$ and $C^{\top} \hat{w}^{0}=P B \hat{w}^{0}$ (see Lemma 2.7 statement 3). Using this in (20) yields

$$
\begin{aligned}
V_{e}(e(0+))-V_{e}(e(0-))=\left(w^{0}-\hat{w}^{0}\right)^{\top}\left[C\left(x_{0}-\hat{x}_{0}\right)+C B\left(w^{0}-\hat{w}^{0}\right)\right]+ \\
+\left(x_{0}-\hat{x}_{0}\right)^{\top} C^{\top}\left(w^{0}-\hat{w}^{0}\right) .
\end{aligned}
$$

Using Theorem 3.8 and Lemma 3.11, it follows that $z^{+}:=C x_{0}+C B w^{0}+F u(0) \in K(D)=\mathcal{Q}_{D}^{*}$ and $\hat{z}^{+}:=C \hat{x}_{0}+C B \hat{w}^{0}+F u(0) \in \mathcal{Q}_{D}^{*}$. Observe that $z^{+}-\hat{z}^{+}=C\left(x_{0}-\hat{x}_{0}\right)+C B\left(w^{0}-\hat{w}^{0}\right)$. Hence, from (21) we obtain

$$
V_{e}(e(0+))-V_{e}(e(0-))=2\left(w^{0}-\hat{w}^{0}\right)^{\top}\left(z^{+}-\hat{z}^{+}\right)-\left(w_{0}-\hat{w}_{0}\right)^{\top} C B\left(w^{0}-\hat{w}^{0}\right)
$$

Since $\mathcal{Q}_{D} \ni w^{0} \perp z^{+} \in \mathcal{Q}_{D}^{*}$ and $\mathcal{Q}_{D} \ni \hat{w}^{0} \perp \hat{z}^{+} \in \mathcal{Q}_{D}^{*}$ (orthogonality follows from Lemma 3.11), we have $\left(w^{0}-\hat{w}^{0}\right)^{\top}\left(z^{+}-\hat{z}^{+}\right)^{\top} \leq 0$. Moreover, since $(D+D)^{\top} w^{0}=(D+D)^{\top} \hat{w}^{0}=0$ it follows that $\left(w^{0}-\hat{w}^{0}\right)^{\top} C B\left(w^{0}-\hat{w}^{0}\right) \geq 0$ due to Lemma 2.7. Hence, from (22) we find that $V_{e}(e(0+)) \leq V_{e}(e(0-))$, which shows that the function $V_{e}$ is non-increasing during jumps at initial time 0 . Similar arguments hold for any other jumps (at times of discontinuity of $F u$ ) since the jumps match a time-shifted initial solution. It now follows from a standard Lyapunov argument that the error dynamics (18) is GES in $e$.

The above theorem shows (under the given hypothesis) that the observer (17) recovers exponentially the state of the LCS (1) based on the output (16), even when the state variable of the system or observer exhibits jumps. Since the jumps in both the observer and the observed plant are triggered by discontinuities in the external signal (due to the low index of the underlying linear system), the time instants of the jumps coincide for the observer and the plant, and this fact is exploited in the proof. In the case of higher-index systems (e.g. mechanical systems with unilateral constraints) this property is lost, which complicates observer design significantly.

5. Stabilization of LCS by dynamic output feedback

\subsection{Stabilization of LCS by state feedback}

In this short subsection we consider the design of a state feedback controller

$$
u(t)=K x(t)
$$

that renders the system (1) GES. The design condition that we use is the following.

Condition 5.1. The matrix $K$ is chosen such that the four-tuple $(A+E K, B, C+F K, D)$ is strictly passive. 
For necessary and sufficient conditions of "passifiability" for the case $D=0$ and single-input singleoutput system (i.e. finding $K$ such that $(A+E K, B, C+F K, 0)$ is strictly passive), we refer the interested reader to $[4,5]$.

Theorem 5.2. Consider the LCS (1) and the state feedback (23) and suppose that the nonredundancy Assumption 2.6 and the design Condition 5.1 are satisfied. Then the closed-loop system (1)-(23) is GES.

In $[11,12]$ it was shown that a sufficient condition for GES of LCS is the strict passivity of the underlying linear system. The above theorem on state feedback design is a direct consequence of that result.

\subsection{Observer-based control}

To design an output-based controller for (1) with (16), we employ an observer-based approach. This means that we apply a state feedback law to the estimate of the state that is produced by an observer. Of course it requires proof to show that this scheme is indeed effective, in the sense that the closed-loop system is well-posed and is globally exponentially stable. The closed-loop system consisting of the system (1), the observer (17) and the controller $u(t)=K \hat{x}(t)$ is

$$
\begin{gathered}
\left(\begin{array}{l}
\dot{x} \\
\dot{e}
\end{array}\right)=\underbrace{\left(\begin{array}{cc}
(A+E K) & -E K \\
0 & A-L G
\end{array}\right)}_{=: A_{c l}}\left(\begin{array}{l}
x \\
e
\end{array}\right)+\underbrace{\left(\begin{array}{cc}
B & 0 \\
B & -B
\end{array}\right)}_{=: B_{c l}}\left(\begin{array}{l}
w \\
\hat{w}
\end{array}\right) \\
\left(\begin{array}{l}
z \\
\hat{z}
\end{array}\right)=\underbrace{\left(\begin{array}{cc}
C+F K & -F K \\
C+F K & -(C+F K)
\end{array}\right)}_{=: C_{c l}}\left(\begin{array}{l}
x \\
e
\end{array}\right)+\underbrace{\left(\begin{array}{cc}
D & 0 \\
0 & D
\end{array}\right)}_{=: D_{c l}}\left(\begin{array}{l}
w \\
\hat{w}
\end{array}\right) \\
0 \leq\left(\begin{array}{l}
z \\
\hat{z}
\end{array}\right) \perp\left(\begin{array}{l}
w \\
\hat{w}
\end{array}\right) \geq 0
\end{gathered}
$$

Theorem 5.3. Consider the system (24), and suppose that the nonredundancy Assumption 2.6 and the design Conditions 4.2 and 5.1 are satisfied.The system (24) has for each initial condition $x_{0}$ and $e_{0}$ a unique global solution in the sense of Definition 3.16. Moreover, the state trajectory $x=\mathrm{x}_{\text {reg }}$ is continuous with the possible exception of a jump discontinuity at time 0 .

Proof. According to Theorem 3.17 it is sufficient to show that the transfer matrix $H(s):=C_{c l}(s I-$ $\left.A_{c l}\right)^{-1} B_{c l}+D_{c l}$ corresponding to (24) is totally of index 1 and is a P-matrix for sufficiently large values of $s$. As $H(s)$ is totally of index 1 if and only if the rational matrix $\bar{H}(s):=D_{c l}+s^{-1} C_{c l} B_{c l}$ is totally of index 1 (see Lemma 3.6), we study $\bar{H}(s)$, which is of the form $\left(\begin{array}{cc}\bar{H}_{11}(s) & \bar{H}_{12}(s) \\ 0 & \bar{H}_{22}(s)\end{array}\right)$ due to the shape of the matrices $D_{c l}$ and $C_{c l} B_{c l}=\left(\begin{array}{cc}C B & F K B \\ 0 & (C+F K) B\end{array}\right)$. The decomposition of the matrices is in accordance with the vector decomposition $\left(w^{\top} \hat{w}^{\top}\right)^{\top}$ and $\left(z^{\top} \hat{z}^{\top}\right)^{\top}$ in (24b). Consider now the principal submatrix for $J \subseteq\{1, \ldots, 2 m\}$, which is given by

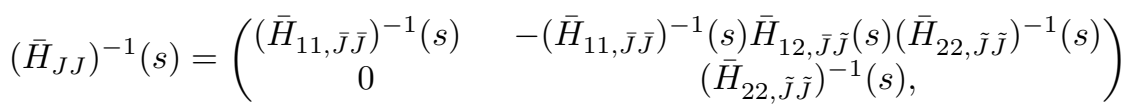

where $\bar{J}$ and $\tilde{J}$ are subsets of $\bar{m}=\{1, \ldots, m\}$ such that $\bar{J} \cup\{i+m \mid i \in \tilde{J}\}=J$. Since $\bar{H}_{11}(s)=D+C B s^{-1}$, we have due to Assumption 2.6, Condition 4.2 and Theorem 3.14 that $\bar{H}_{11}(s)$ 
is totally of index 1. Due to Assumption 2.6, Condition 5.1 and Theorem 3.14, we also have that $\bar{H}_{22}(s)=D+(C+F K) B s^{-1}$ is totally of index 1 . Hence, $s^{-1} \bar{H}_{11, \bar{J} \bar{J}}^{-1}(s)$ and $s^{-1} \bar{H}_{22, \tilde{J} \tilde{J}}^{-1}(s)$ are proper. As $\bar{H}_{12, \bar{J} \tilde{J}}(s)$ is strictly proper, it follows that $s^{-1}\left(\bar{H}_{11, \bar{J} \bar{J}}\right)^{-1}(s) \bar{H}_{12, \bar{J} \tilde{J}}(s)\left(\bar{H}_{22, \tilde{J} \tilde{J}}\right)^{-1}(s)$ is proper as well. This shows that $s^{-1}\left(\bar{H}_{J J}\right)^{-1}(s)$ is proper. Hence, $\bar{H}(s)$ and thus $H(s)$ are totally of index 1.

Given the triangular form of $\bar{H}(s), \bar{H}(s)$ is a $P$-matrix if and only if its diagonal blocks $\bar{H}_{11}(s)=$ $D+C B s^{-1}$ and $\bar{H}_{22}(s)=D+(C+F K) B s^{-1}$ are so. This is most easily seen by using that $\operatorname{det}\left(\begin{array}{cc}R_{1} & R_{2} \\ 0 & R_{3}\end{array}\right)$ is equal to $\operatorname{det}\left(R_{1}\right) \cdot \operatorname{det}\left(R_{3}\right)$ and each principal submatrix of $\bar{H}(s)$ has a block diagonal structure with principal submatrices of $\bar{H}_{11}(s)$ and $\bar{H}_{22}(s)$ on the diagonal. Given the hypothesis of the theorem we can use Theorem 3.14 to conclude that both $\bar{H}_{11}(s)=D+s^{-1} C B$ and $\bar{H}_{22}(s)=D+s^{-1}(C+F K) B$ are P-matrices for sufficiently large $s$. This means that $H(s)$ is a $P$-matrix for sufficiently large $s$. Invoking now Theorem 3.17 completes the proof. Since there are no external inputs, the only point at which impulses can occur (and consequently, discontinuities in the state trajectory) is at time 0 .

Now we can show GES of the closed-loop LCS (24).

Theorem 5.4 (Stabilizing observer-based controller) If the nonredundancy Assumption 2.6 and the design Conditions 4.2 and 5.1 hold, then the closed-loop LCS (24) is GES.

Proof. The global existence (and uniqueness) of solutions given an initial condition has already been established, see Theorem 5.3. Let $P_{e}$ and $\varepsilon>0$ be a solution to (19) and $P_{x}$ and $\varepsilon>0$ be a solution to

$$
P_{x}=P_{x}^{\top}>0 \text { and }\left(\begin{array}{cc}
(A+E K)^{\top} P_{x}+P_{x}(A+E K)+\varepsilon P_{x} & P_{x} B-(C+F K)^{\top} \\
B^{\top} P_{x}-(C+F K) & -\left(D+D^{\top}\right)
\end{array}\right) \leq 0 .
$$

We will consider the candidate Lyapunov function $V_{\beta}(x, e):=V_{x}(x)+\beta V_{e}(e)$ for some $\beta>0$, where $V_{x}(x)=x^{\top} P_{x} x$ and $V_{e}(e)=e^{\top} P_{e} e$. According to Theorem 5.3 the system (24) has only discontinuities in the state at the initial time 0 . Since $H_{J}(s)$ is invertible as a rational matrix for each mode $J$, we obtain from [19] that there exist linear spaces $\mathcal{V}_{J}$ and $\mathcal{T}_{J}$ such that $\mathcal{V}_{J} \oplus \mathcal{T}_{J}=\mathbb{R}^{2 n}$. Moreover, [19] shows that the reset in mode $J$ corresponding to the initial solution in mode $J$ satisfies $\left(\begin{array}{c}x^{+} \\ e^{+}\end{array}\right)=P_{J}\left(\begin{array}{l}x_{0} \\ e_{0}\end{array}\right)$, where $P_{J}$ is the projection onto $\mathcal{V}_{J}$ along $\mathcal{T}_{J}$. Since the number of modes is finite, this implies that there exists a constant $\rho>0$ such that $\left\|\left(\begin{array}{c}x^{+} \\ e^{+}\end{array}\right)\right\| \leq \rho\left\|\left(\begin{array}{l}x_{0} \\ e_{0}\end{array}\right)\right\|$. Hence, we have a bound on the jump that the state of the system (24) might take at time 0 .

Next we consider the time derivative of the function $V_{\beta}$ along trajectories of the LCS (24). First, we compute the derivative of $V_{x}$ :

$$
\begin{aligned}
\dot{V}_{x}= & -e^{\top} K^{\top} E^{\top} P_{x} x+w^{\top} B^{\top} P_{x} x+x^{\top}(A+E K)^{\top} P_{x} x+x^{\top} P_{x}(A+E K) x+ \\
& +x^{\top} P_{x} B w-x^{\top} P_{x} E K e \\
= & \left(\begin{array}{c}
x \\
w
\end{array}\right)^{\top}\left(\begin{array}{cc}
(A+E K)^{\top} P_{x}+P_{x}(A+E K) & P_{x} B \\
B^{\top} P_{x} & 0
\end{array}\right)\left(\begin{array}{c}
x \\
w
\end{array}\right)-e^{\top} K^{\top} E^{\top} P_{x} x-x^{\top} P_{x} E K e \\
= & \left(\begin{array}{c}
x \\
w
\end{array}\right)^{\top}\left(\begin{array}{cc}
(A+E K)^{\top} P_{x}+P_{x}(A+E K) & P_{x} B-(C+F K)^{\top} \\
B^{\top} P_{x}-(C+F K) & -\left(D+D^{\top}\right)
\end{array}\right)\left(\begin{array}{c}
x \\
w
\end{array}\right)+ \\
& +x^{\top}(C+F K)^{\top} w+w^{\top}(C+F K) x+w^{\top}\left(D+D^{\top}\right) w-e^{\top} K^{\top} E^{\top} P_{x} x-x^{\top} P_{x} E K e
\end{aligned}
$$




$$
\begin{aligned}
& \leq-\varepsilon x^{\top} P_{x} x+(z+F K e)^{\top} w+w^{\top}(z+F K e)-2 x^{\top} P_{x} E K e \\
& =-\varepsilon x^{\top} P_{x} x+e^{\top} K^{\top} F^{\top} w+w^{\top} F K e-2 x^{\top} P_{x} E K e,
\end{aligned}
$$

where we used in the latter equality that $z^{\top} w=0$.

In a similar way as in the proof of Theorem 4.5, we can derive that $V_{e}$ satisfies $\dot{V}_{e} \leq-\varepsilon V_{e}$, which implies that the time derivative of $V_{\beta}$ along trajectories satisfies the inequality

$$
\dot{V}_{\beta}(x, e) \leq-\varepsilon x^{\top} P_{x} x+e^{\top} K^{\top} F^{\top} w+w^{\top} F K e-2 x^{\top} P_{x} E K e-\beta \varepsilon e^{\top} P_{e} e .
$$

Since $w$ is a complementarity variable of the LCS (24), for each $t \geq 0$ there is a $\delta>0$ and $J \subseteq\{1, \ldots, 2 m\}$ (depending on $t$ ) such that $w$ is determined on $[t, t+\delta$ ) by the mode- $J$ dynamics of (24), i.e. it satisfies

$$
\begin{aligned}
& \left(\begin{array}{l}
\dot{x} \\
\dot{e}
\end{array}\right)=A_{c l}\left(\begin{array}{l}
x \\
e
\end{array}\right)+B_{c l}\left(\begin{array}{l}
w \\
\hat{w}
\end{array}\right) ;\left(\begin{array}{l}
z \\
\hat{z}
\end{array}\right)=C_{c l}\left(\begin{array}{l}
x \\
e
\end{array}\right)+D_{c l}\left(\begin{array}{l}
w \\
\hat{w}
\end{array}\right) \\
& z_{i}=0, i \in \bar{J}, \hat{z}_{i}=0, i \in \tilde{J}, w_{i}=0, i \in \bar{J}^{c}, \hat{w}_{i}=0, i \in \tilde{J}^{c}
\end{aligned}
$$

where $\bar{J}$ and $\tilde{J}$ are subsets of $\{1, \ldots, m\}$ such that $\bar{J} \cup\{i+m \mid i \in \tilde{J}\}=J$. According to Theorem 3.10 in [19], the fact that $H_{J J}(s)$ is invertible as a rational matrix implies that there is a matrix $R_{J}$ such that $w=R_{J}\left(x^{\top} e^{\top}\right)^{\top}$ (i.e. we solved (27) for $w$ in terms of $x$ and $e$ ). Since $w$ is a solution at every time $t$ to (27) for some $J$ and there are finitely many modes, it follows that there is a $\gamma>0$ such that $\|w\| \leq \gamma(\|x\|+\|e\|)$. From the Cauchy-Schwarz inequality applied to (26) and from the fact that $x^{\top} P_{x} x \geq \alpha_{x}\|x\|^{2}$ and $e^{\top} P_{x} e \geq \alpha_{e}\|e\|^{2}$ for some positive constants $\alpha_{x}$ and $\alpha_{e}$, it follows that there exist constants $\eta$ and $\theta$ such that

$\dot{V}_{\beta}(x, e) \leq-\varepsilon \alpha_{x}\|x\|^{2}-\beta \varepsilon \alpha_{e}\|e\|^{2}+\eta\|e\|^{2}+2 \theta\|e\|\|x\|=-\left(\begin{array}{l}\|x\| \\ \|e\|\end{array}\right)^{\top}\left(\begin{array}{cc}\varepsilon \alpha_{x} & \theta \\ \theta & \beta \varepsilon \alpha_{e}-\eta\end{array}\right)\left(\begin{array}{l}\|x\| \\ \|e\|\end{array}\right)$.

Since for sufficiently large $\beta$ the matrix $\left(\begin{array}{cc}\varepsilon \alpha_{x} & \theta \\ \theta & \beta \varepsilon \alpha_{e}-\eta\end{array}\right)$ is positive definite, it follows that $V_{\beta}$ is a Lyapunov function for (24). This proves GES.

Theorem 5.4 provides a formal justification of the separate design of the state feedback law and the observer.

Remark 5.5. Of course, one could attempt a direct approach, in the sense of designing the state feedback and observer gains $K$ and $L$ simultaneously in order that $\left(A_{c l}, B_{c l}, C_{c l}, D_{c l}\right)$ satisfy the conditions of Theorem 5.4. Here, we followed an observer-based approach using a state feedback controller for which the gain $K$ is designed separately satisfying the conditions of Theorem 5.2, and an observer with gain $L$ is constructed satisfying the conditions of Theorem 4.5. The closed-loop system (24) is then guaranteed to be GES under the hypothesis of Theorem 5.4. On the basis of stability results that are currently available, the direct approach would require $\left(A_{c l}, B_{c l}, C_{c l}, D_{c l}\right)$ to be strictly passive, while for our observer-based control approach we have separate passivity requirements for state feedback and observer gain that do not necessarily impose $\left(A_{c l}, B_{c l}, C_{c l}, D_{c l}\right)$ to be strictly passive. 


\section{Extensions}

Instead of the observer as in (17) we may consider the extended observer, which is of the form

$$
\begin{gathered}
\dot{\hat{x}}(t)=A \hat{x}(t)+B \hat{w}(t)+E u(t)+L(y(t)-\hat{y}(t)) \\
\hat{z}(t)=C \hat{x}(t)+D \hat{w}(t)+F u(t)+M(y(t)-\hat{y}(t)) \\
0 \leq \hat{z}(t) \perp \hat{w}(t) \geq 0 \\
\hat{y}(t)=G \hat{x}(t),
\end{gathered}
$$

where we now have two observer gains $(L$ and $M)$. The design condition 4.2 is then modified as follows.

Condition 6.1. The matrices $L$ and $M$ are chosen such that $(A-L G, B, C-M G, D)$ is strictly passive.

In a similar way as in the proof of Theorem 4.5 we can obtain the following result.

Theorem 6.2. Consider the error dynamics (18) with (1) and (28) for input $u \in \mathcal{P B}^{k}$ such that Assumption 2.6, Assumption 4.1 and Condition 6.1 hold. Then the error dynamics is GES.

Using the extended observer instead of the basic observer (i.e. observer (17)) adds a degree of freedom $M$ for the observer-based control design (under the assumption that $F=0$ ). The usefulness of this extra degree of freedom is demonstrated by the following example for which $(A-L G, B, C, D)$ cannot be made strictly passive, while $(A-L G, B, C-M G, D)$ can. Hence, an extended observer (28) that recovers the state exponentially can be found using the developed theory, but a basic observer can not.

Example 6.3. Consider the LCS (1) without inputs (so $E$ and $F$ absent) and with

$$
A=\left(\begin{array}{cc}
1 & 0 \\
0 & -1
\end{array}\right) ; B=\left(\begin{array}{ll}
1 & 0
\end{array}\right)^{\top} ; C=\left(\begin{array}{ll}
0 & 1
\end{array}\right) ; D=0 ; G=\left(\begin{array}{ll}
1 & 0
\end{array}\right) .
$$

If we try to find a matrix $L$ such that $(A-L G, B, C, D)$ is strictly passive, we have to satisfy

$$
(A-L G)^{\top} P+P(A-L G)<0, P>0 \text { and } B^{\top} P=C
$$

due to (11) as $D=0$. Since the condition $B^{\top} P=C$ requires $\left(\begin{array}{ll}1 & 0\end{array}\right) P=\left(\begin{array}{ll}0 & 1\end{array}\right)$, we can conclude that the first row of $P$ must then be equal to $\left(\begin{array}{ll}0 & 1\end{array}\right)$, which obstructs the positive definiteness of $P$. Hence, one cannot make $(A-L G, B, C, D)$ strictly passive by suitable choice of $L$. Consequently, we will not be able to find a basic observer with the results outlined in this paper. However, with $L=\left(\begin{array}{ll}2 & 0\end{array}\right)^{\top}$ and $M=-1$, we get $A-L G=-I$ and $C-M G=\left(\begin{array}{ll}1 & 1\end{array}\right)$. Since the matrix $P=\left(\begin{array}{ll}1 & 1 \\ 1 & 2\end{array}\right)$ is positive definite and satisfies $(A-L G)^{\top} P+P(A-L G)<0$ and $B^{\top} P=C-M G$, the four-tuple $(A-L G, B, C-M G, D)$ is strictly passive and an extended observer can be designed based on Theorem 6.2.

Considering the observer-based controller consisting of (28) and $u(t)=K \hat{x}(t)$ in closed loop with (1) yields

$$
\left(\begin{array}{l}
\dot{x} \\
\dot{e}
\end{array}\right)=\underbrace{\left(\begin{array}{cc}
(A+E K) & -E K \\
0 & A-L G
\end{array}\right)}_{=: A_{c l}}\left(\begin{array}{l}
x \\
e
\end{array}\right)+\underbrace{\left(\begin{array}{cc}
B & 0 \\
B & -B
\end{array}\right)}_{=: B_{c l}}\left(\begin{array}{l}
w \\
\hat{w}
\end{array}\right)
$$




$$
\begin{gathered}
\left(\begin{array}{l}
z \\
\hat{z}
\end{array}\right)=\underbrace{\left(\begin{array}{cc}
C+F K & -F K \\
C+F K & -(C+F K-M G)
\end{array}\right)}_{=: C_{c l}}\left(\begin{array}{l}
x \\
e
\end{array}\right)+\underbrace{\left(\begin{array}{cc}
D & 0 \\
0 & D
\end{array}\right)}_{=: D_{c l}}\left(\begin{array}{l}
w \\
\hat{w}
\end{array}\right) \\
0 \leq\left(\begin{array}{l}
z \\
\hat{z}
\end{array}\right) \perp\left(\begin{array}{l}
w \\
\hat{w}
\end{array}\right) \geq 0
\end{gathered}
$$

Following similar reasoning as in the proof of Theorem 5.4, we can prove the following theorem:

Theorem 6.4. Consider the closed-loop LCS (24). If the nonredundancy Assumption 2.6 and the design Conditions 5.1 and 6.1 are satisfied and $F=0$, then the LCS (24) is GES.

Note that for the observer-based stabilization result as formulated in the latter theorem, we require that $F=0$ (no direct feedthrough of external input $u$ into complementarity conditions). The reason why this assumption helps is that under this condition the matrix $\bar{H}(s)=D_{c l}+s^{-1} C_{c l} B_{c l}$ has a block triangular structure. This is easy seen if we write down $C_{c l} B_{c l}$ (note that $D_{c l}$ is always diagonal), which in the general case is equal to $C_{c l} B_{c l}=\left(\begin{array}{cc}C B & F K B \\ M G B & (C+F K-M G) B\end{array}\right)$. Clearly, for $F=0$ we have a lower block triangular form as in the basic observer case $(M=0)$, and the proof of Theorem 5.3 can be applied mutatis mutandis. However, in the general case $M \neq 0$ and $F \neq 0$ we do not in general have a triangular form as exploited in the proof of Theorem 5.3, and there is no straightforward way to adapt the proof. Note that a block triangular structure is also key to the proof of closed-loop stability for the observer-based scheme in the classical linear systems case, as discussed in the Introduction. To establish the well-posedness of the closed-loop system in absence of the triangular structure is at present an open problem. However, note that part of the stability proof of Theorem 5.4 (where we establish that the time derivative of $V_{\beta}$ is negative definite for sufficiently large $\beta$ ) is still valid even without the assumption that either $M=0$ or $F=0$.

\section{Conclusions}

This paper has demonstrated that the classical separation technique for stabilization by output feedback can be extended, under certain conditions, from linear systems to linear complementarity systems (LCS). LCS arise in many applications and enjoy an appealing structure; at the same time, they are hybrid systems that show intrinsic properties such as the presence of state jumps, mode dynamics described by DAEs, and regions ("invariants") for certain modes being lower dimensional, which preclude application of various observer and controller design methods that have been proposed in the literature on hybrid systems. Even the well-posedness issue (existence and uniqueness of solutions given an initial condition and input signals) is nontrivial due to the set-valuedness of the complementarity conditions.

The success of a stabilization scheme would be in doubt if the well-posedness of the closed-loop system would not be ensured. Therefore, we first presented novel conditions for the well-posedness of LCS using low-index conditions; this has allowed us to avoid imposing passitivity requirements on the closed-loop system as a whole. As part of the construction we considered observer design and presented sufficient conditions for the the estimated state to recover the state of the system at an exponential rate, even in the presence of state jumps. We then constructed a dynamic output feedback controller by applying a state feedback law to the estimated state produced by the observed, and we 
justified this construction by showing that the resulting closed-loop system is well-posed and globally exponentially stable.

An item of future research is the extended observer scheme mentioned in the previous section. Wellposedness of the observer and exponential recovery of the state could be proven for this scheme as well. In addition, in case there is no direct feedthrough of the external input into the complementarity conditions (i.e. $F=0$ ), the observer-based controller is well-posed and stabilizes the original LCS. Whether this is also true when $F \neq 0$ and the extended observer is used is presently an open problem; curiously, the main difficulty appears to reside in showing the well-posedness of the resulting closedloop system, rather than in the stability proof.

This paper has shown that successful design of observers and stabilization schemes based on output feedback is possible for certain classes of hybrid systems, even in situations where the observer mode may well be different from the state mode, and in the presence of state jumps. However, we only considered situations in which jumps in the state and in the observer occur at the same time. This is due to the fact that we have restricted ourselves to low-index LCS in this paper; in such systems jumps occur only at the initial time or when they are externally induced. Situations in which state jumps are internally induced are of interest as well. In these situations it would seem difficult to ensure that jumps of the true state and of the estimated state occur at the same time. Examples of situations of this type are provided by higher-index LCS such as arise in the description of mechanical systems subject to unilateral constraints. Design of observers and output feedback stabilizers in these cases stands as a major challenge.

\section{Appendix}

Proof of Lemma 3.6. We achieve the proof of this lemma in the following order.

$$
\begin{aligned}
1 & \Leftrightarrow 2 \\
2 & \Rightarrow 3 \\
3 & \Rightarrow 4 \\
4 & \Rightarrow 2
\end{aligned}
$$

We denote the rational matrices $H^{0}+H^{1} s^{-1}$ and $H^{2} s^{-2}+H^{3} s^{-3}+\cdots$ by $H_{\text {low }}(s)$ and $H_{\text {high }}(s)$, respectively.

$1 \Rightarrow 2$ : Since $H(s)$ is of index 1 , it is invertible as a rational matrix. Thus, we have

$$
\left[H_{\text {low }}(s)+H_{\text {high }}(s)\right] H^{-1}(s)=I .
$$

Since $H(s)$ is of index $1, s^{-2} H^{-1}(s)$ is strictly proper and so is $H_{h i g h}(s) H^{-1}(s)$. It follows from (31) that

$$
\lim _{s \rightarrow \infty} H_{\text {low }}(s) H^{-1}(s)=I .
$$

Therefore, $H_{\text {low }}(s) H^{-1}(s)$ is biproper. This means that $H_{l o w}(s)$ is also invertible as a rational matrix. On the other hand, (32) can be rewritten as

$$
\lim _{s \rightarrow \infty} s H(s) s^{-1} H_{l o w}^{-1}(s)=I .
$$


Since $H(s)$ is of index $1, \lim _{s \rightarrow \infty} s^{-1} H^{-1}(s)$ is well-defined. Left multiplying (33) by $\lim _{s \rightarrow \infty} s^{-1} H^{-1}(s)$ results in

$$
\lim _{s \rightarrow \infty} s^{-1} H_{l o w}^{-1}(s)=\lim _{s \rightarrow \infty} s^{-1} H^{-1}(s) .
$$

Clearly, this implies that $s^{-1} H_{l o w}^{-1}(s)$ is proper and hence $H_{\text {low }}(s)$ is of index 1 .

$2 \Rightarrow 1:$ Note that

$$
H(s)=H_{\text {low }}(s)\left[I+H_{\text {low }}^{-1}(s) H_{\text {high }}(s)\right] .
$$

Since $H_{\text {low }}(s)$ is of index $1, s^{-2} H_{l o w}^{-1}(s)$ is strictly proper and so is $H_{l o w}^{-1}(s) H_{h i g h}(s)$. Therefore, the second factor on the right hand side of (34) is biproper. Consequently, $H(s)$ is of index 1.

$2 \Rightarrow 3$ : Since $H^{0}+H^{1} s^{-1}$ is of index 1 , the power series expansion of its inverse can be given by

$$
\left(H^{0}+H^{1} s^{-1}\right)^{-1}=N^{-1} s+N^{0}+N^{1} s^{-1}+\cdots .
$$

Note that (35) gives

$$
\begin{gathered}
H^{0} N^{-1}=0 \\
N^{-1} H^{0}=0 \\
N^{-1} H^{1}+N^{0} H^{0}=I .
\end{gathered}
$$

Suppose that $u \in \operatorname{im} H^{0} \cap H^{1}\left(\operatorname{ker} H^{0}\right)$. Then, we have

$$
\begin{aligned}
u & =H^{0} v \\
u & =H^{1} w \\
0 & =H^{0} w
\end{aligned}
$$

for some $v$ and $w$. It follows that

$$
\left(H^{0}+H^{1} s^{-1}\right) w \stackrel{(41)}{=} s^{-1} H^{1} w \stackrel{(40)}{=} s^{-1} u \stackrel{(39)}{=} s^{-1} H^{0} v .
$$

Then, (35) yields

$$
w=\left(N^{-1} s+N^{0}+N^{1} s^{-1}+\cdots\right) H^{0} v s^{-1} \stackrel{(37)}{=} N^{0} H^{0} v s^{-1}+\cdots .
$$

Since the right hand side of the above equation is strictly proper, $w$ is zero and so is $u$ due to (40). Hence, im $H^{0} \cap H^{1}\left(\operatorname{ker} H^{0}\right)=\{0\}$. We have

- (36) $\Rightarrow \operatorname{im} N^{-1} \subseteq \operatorname{ker} H^{0}$,

- $(38) \Rightarrow\left(v \in \operatorname{ker} \bar{H}^{0} \Rightarrow v \in \operatorname{im} N^{-1}\right) \Rightarrow \operatorname{ker} H^{0} \subseteq \operatorname{im} N^{-1}$.

Obviously, these statements imply that ker $H^{0}=\operatorname{im} N^{-1}$. Thus, one gets $H^{1}\left(\operatorname{ker} H^{0}\right)=\operatorname{im} H^{1} N^{-1}$. Suppose that $u \in\left(\operatorname{im} H^{0}+H^{1}\left(\operatorname{ker} H^{0}\right)\right)^{\perp}$, i. e.,

$$
\begin{gathered}
u^{T} H^{0}=0 \\
u^{T} H^{1} N^{-1}=0 .
\end{gathered}
$$


Then, we get

$$
\begin{aligned}
u^{T} & \stackrel{(35)}{=} u^{T}\left(H^{0}+H^{1} s^{-1}\right)\left(N^{-1} s+N^{0}+N^{1} s^{-1}+\cdots\right) \\
& \stackrel{(42)}{=} u^{T} H^{1} s^{-1}\left(N^{-1} s+N^{0}+N^{1} s^{-1}+\cdots\right) \\
& \stackrel{(43)}{=} u^{T} H^{1} N^{0} s^{-1}+\cdots .
\end{aligned}
$$

The fact that the right hand side of the above equation is strictly proper implies that $u$ is zero. Hence, $\operatorname{im} H^{0}+H^{1}\left(\operatorname{ker} H^{0}\right)=\mathbb{R}^{l}$. It follows from (i) and (ii) that im $H^{0} \oplus H^{1}\left(\operatorname{ker} H^{0}\right)=\mathbb{R}^{l}$.

$3 \Rightarrow 4$ : Let $Q \in \mathbb{R}^{q \times l}$ be such that $\operatorname{ker} Q=\operatorname{im} H^{0}$. Take any $P \in \mathbb{R}^{(l-q) \times l}$ such that $\operatorname{col}(P, Q)$ is nonsingular. Suppose that

$$
\left[\begin{array}{l}
P H^{0} \\
Q H^{1}
\end{array}\right] x=0
$$

for some $x \in \mathbb{R}^{l}$. Since $\operatorname{ker} Q=\operatorname{im} H^{0}$, we have $\operatorname{col}(P, Q) H^{0} x=0$ from (44). This implies that, $H^{0} x=0$, i.e., $x \in$ ker $H^{0}$. Hence, $H^{1} x \in H^{1}$ (ker $\left.H^{0}\right)$. On the other hand, (44) also yields $H^{1} x \in \operatorname{ker} Q=\operatorname{im} H^{0}$. Therefore, $H^{1} x \in \operatorname{im} H^{0} \cap H^{1}\left(\operatorname{ker} H^{0}\right)$. It follows from the hypothesis that $H^{1} x=0$. Note that

$$
\operatorname{dim}\left(\operatorname{im} H^{0}\right)+\operatorname{dim}\left(H^{1}\left(\operatorname{ker} H^{0}\right)\right)=l=\operatorname{dim}\left(\operatorname{im} H^{0}\right)+\operatorname{dim}\left(\operatorname{ker} H^{0}\right) .
$$

Thus, we have $\operatorname{dim}\left(H^{1}\left(\operatorname{ker} H^{0}\right)\right)=\operatorname{dim}\left(\operatorname{ker} H^{0}\right)$. In other words, $\operatorname{ker}\left(\left.H^{1}\right|_{\operatorname{ker} H^{0}}\right)=\{0\}$. It follows from $H^{0} x=H^{1} x=0$ that $x=0$ and hence $\operatorname{col}\left(P H^{0}, Q H^{1}\right)$ is nonsingular.

$4 \Rightarrow 2:$ Note that

$$
\begin{aligned}
H^{0}+s^{-1} H^{1} & =\left(\begin{array}{l}
P \\
Q
\end{array}\right)^{-1}\left(\begin{array}{l}
P \\
Q
\end{array}\right)\left(H^{0}+s^{-1} H^{1}\right)=\left(\begin{array}{c}
P \\
Q
\end{array}\right)^{-1}\left[\left(\begin{array}{c}
P H^{0} \\
0
\end{array}\right)+s^{-1}\left(\begin{array}{c}
P H^{1} \\
Q H^{1}
\end{array}\right)\right] \\
& =\left(\begin{array}{c}
P \\
Q
\end{array}\right)^{-1}\left(\begin{array}{cc}
I & 0 \\
0 & s^{-1} I
\end{array}\right)\left[\left(\begin{array}{c}
P H^{0} \\
Q H^{1}
\end{array}\right)+s^{-1}\left(\begin{array}{c}
P H^{1} \\
0
\end{array}\right)\right] .
\end{aligned}
$$

It follows from the hypothesis that the matrix $\left(\begin{array}{c}P H^{0} \\ Q H^{1}\end{array}\right)$ is nonsingular that $H^{0}+s^{-1} H^{1}$ is of index 1 .

Proof of Lemma 3.11. In this proof we will use the one-to-one correspondence between the solutions of RCP and initial solutions to LCS as stated in Proposition 3.10. Since the Laplace transform $\hat{u}(s)$ is strictly proper and $\hat{w}(s)$ is proper, their power series expansion around infinity can be given by

$$
\begin{gathered}
\hat{w}(s)=w^{0}+w_{1} s^{-1}+w_{2} s^{-2}+\cdots \\
\hat{u}(s)=u_{1} s^{-1}+u_{2} s^{-2}+\cdots .
\end{gathered}
$$

Then, we get based on (12) that

$$
\hat{z}(s)=D w^{0}+\left(C x_{0}+F u(0)+C B w^{0}+D w_{1}\right) s^{-1}+\ldots
$$

As $\hat{w}(s)$ is a solution to $\operatorname{RCP}\left(x_{0}, \hat{u}(s)\right)$, it follows by inspection of the constant terms and using (46) and (47) that $w^{0} \geq 0, D w^{0} \geq 0$ and $w^{0 T} D w^{0}=0$ and thus $w^{0} \in \mathcal{Q}_{D}$. Clearly, we also have that 
$\mathbf{z}_{i m p}=D w^{0} \delta_{0}$ due to property (47) by using the inverse Laplace transform. Similarly, using (13a) and strict properness of $\hat{u}(s)$ and $(s I-A)^{-1}$, we obtain $\mathrm{x}_{i m p}=0$. This proves (i).

Moreover, since $\hat{w}(s)$ is a solution to $\operatorname{RCP}\left(x_{0}, \hat{u}(s)\right)$, we also have

$$
\begin{gathered}
w^{0}+w_{1} \sigma^{-1} \geq 0 \\
C x_{0}+F u(0)+C B w^{0}+D\left(w^{0}+w_{1} \sigma^{-1}\right) \geq 0 \\
\left(w^{0}+w_{1} \sigma^{-1}\right)^{\top}\left[C x_{0}+F u(0)+C B w^{0}+D\left(w^{0}+w_{1} \sigma^{-1}\right)\right]=0
\end{gathered}
$$

for all sufficiently large $\sigma$. Therefore, $\operatorname{LCP}\left(C x_{0}+F u(0)+C B w^{0}, D\right)$ is solvable. This means that $C x_{0}+F u(0)+C B w^{0} \in K(D)$, thereby proving (ii).

Furthermore, by inspecting only the $s^{-1}$ terms in $\hat{w}^{\top}(s) \hat{z}(s)=0$ we obtain

$$
w^{0 T}\left(C x_{0}+F u(0)+C B w^{0}+D w_{1}\right)+w_{1}^{\top} D w^{0}=0 .
$$

This yields

$$
w^{0 T}\left(C x_{0}+F u(0)+C B w^{0}\right)+w_{1}^{\top}\left[D+D^{\top}\right] w^{0}=0 .
$$

Since $w^{0} \in \mathcal{Q}_{D}$ and the hypothesis, we obtain $\left(D+D^{\top}\right) w^{0}=0$ and thus the last term in the right-hand side above is zero, which shows (iii).

Proof of Lemma 3.12. (i): Since $D+C(\sigma I-A)^{-1} B$ is a P-matrix for all sufficiently large $\sigma$, [24, Theorem 4.1] and Theorem 2.1 imply that $\operatorname{RCP}\left(x_{0}, \hat{u}(s)\right)$ has a unique solution for all $x_{0} \in \mathbb{R}^{n}$ and for all $\hat{u}(s) \in \mathbb{R}^{p}(s)$. Due to Proposition 3.10 this establishes (i).

(ii): Let $\hat{w}(s)$ be the unique solution of $\operatorname{RCP}\left(x_{0}, \hat{u}(s)\right)$. For the 'only if' part, suppose that $\hat{w}(s)$ is strictly proper. Let the power series expansion around infinity of $\hat{w}(s)$ and $\hat{u}(s)$ be of the form

$$
\begin{gathered}
\hat{w}(s)=w_{1} s^{-1}+w_{2} s^{-2}+\ldots \\
\hat{u}(s)=u_{1} s^{-1}+u_{2} s^{-2}+\ldots
\end{gathered}
$$

Define

$$
\hat{z}(s)=C(s I-A)^{-1} x_{0}+\left[D+C(s I-A)^{-1} B\right] \hat{w}(s)+\left[F+C(s I-A)^{-1} E\right] \hat{u}(s) .
$$

By substituting (51) into the above equation, we get

$$
\hat{z}(s)=\left(C x_{0}+F u_{1}+D w_{1}\right) s^{-1}+\left(C A x_{0}+C E u_{1}+F u_{2}+C B w_{1}+D w_{2}\right) s^{-2}+\ldots .
$$

It follows from the formulation of $\operatorname{RCP}\left(x_{0}, \hat{u}(s)\right)$ that

$$
\begin{gathered}
w_{1}^{\top}\left(C x_{0}+F u_{1}+D w_{1}\right)=0 \\
w_{1} \geq 0 \text { and } C x_{0}+F u_{1}+D w_{1} \geq 0 .
\end{gathered}
$$

Consequently, $\operatorname{LCP}\left(C x_{0}+F u_{1}, D\right)$ is solvable. Then, it follows that $C x_{0}+F w(0) \in K(D)$ since $w_{1}=w(0)$. To show the 'if' part, suppose that $C x_{0}+F w(0) \in K(D)$. This means that $\operatorname{LCP}\left(C x_{0}+F w(0), D\right)$ is solvable. Let $\bar{w}$ be a solution of $\operatorname{LCP}\left(C x_{0}+F u(0), D\right)$. It is clear that $\sigma^{-1} \bar{w}$ solves $\operatorname{LCP}\left(\sigma^{-1}\left(C x_{0}+F u(0)\right), D\right)$ for all $\sigma>0$. Then, it also solves $\operatorname{LCP}\left(\sigma^{-1}\left(C x_{0}+F u(0)-\right.\right.$ $\left.\left.C(\sigma I-A)^{-1} B \bar{w}\right), G(\sigma)\right)$. Since $\hat{w}(s)$ is the unique solution to $\operatorname{RCP}\left(x_{0}, \hat{u}(s)\right)$, for sufficiently large $\sigma$ the vector $\hat{w}(\sigma)$ solves $\operatorname{LCP}\left(C(\sigma I-A)^{-1} x_{0}+\left[F+C(\sigma I-A)^{-1} E\right] \hat{u}(\sigma), G(\sigma)\right)$ (see also [24]). Exploiting now Lemma 2.2 leads to

$$
\begin{aligned}
\left\|\hat{w}(\sigma)-\sigma^{-1} \bar{w}\right\| \leq d(G(\sigma)) \| & C(\sigma I-A)^{-1} x_{0}+\left[F+C(\sigma I-A)^{-1} E\right] \hat{u}(\sigma) \\
& -\sigma^{-1}\left(C x_{0}+F u_{1}-C(\sigma I-A)^{-1} B \bar{w}\right) \|
\end{aligned}
$$


for all sufficiently large $\sigma$. Since $G(s)$ is totally of index 1, according to Lemma 3.7 there is an $\alpha>0$ such that $d(G(\sigma)) \leq \alpha \sigma$ for all sufficiently large $\sigma$. Note that there exists $\beta>0$ such that the final factor of the right hand side is less than $\beta \sigma^{-2}$ for all sufficiently large $\sigma$. Therefore, (52) yields

$$
\left\|\hat{w}(\sigma)-\sigma^{-1} \bar{w}\right\| \leq \alpha \beta \sigma^{-1}
$$

for all sufficiently large $\sigma$. This implies that $\hat{w}(s)$ is strictly proper.

\section{REFERENCES}

1. V. Acary and B. Brogliato. Numerical Methods for Nonsmooth Dynamical Systems, volume 35 of Lecture Notes in Applied and Computational Mechanics. Springer, 2008.

2. A. Alessandri and P. Coletta. Design of Luenberger observers for a class of hybrid linear systems. In M.D. di Benedetto and A.L. Sangiovanni-Vincentelli, editors, Proc. 4th Intern. Workshop on Hybrid Systems: Computation and Control (Rome, Italy), LNCS 2034, pages 7-18. Springer, 2001.

3. M. Arcak. Certainty-equivalence output-feedback design with circle-criterion observers. IEEE Trans. Automatic Control, 50:905 - 909, 2005

4. M. Arcak and P. Kokotović. Feasibility conditions for circle criterion designs. Syst. Control Lett., 42(5):405-412, 2001.

5. M. Arcak and P. Kokotović. Observer based control of systems with slope-restricted nonlinearities. IEEE Trans. Automatic Control, 46(7):1146-1150, 2001.

6. A. Bemporad and M. Morari. Control of systems integrating logic, dynamics, and constraints. Automatica, 35:407-427, 1999.

7. M.S. Branicky, V.S. Borkar, and S.K. Mitter. A unified framework for hybrid control: model and optimal control theory. IEEE Trans. Automatic Control, 43(1):31-45, 1998

8. B. Brogliato. Some perspectives on the analysis and control of complementarity systems. IEEE Trans Automatic Control, 48:918 - 935, 2003.

9. B. Brogliato, A. Daniilidis, C. Lemaréchal, and V. Acary. On the equivalence between complementarity systems, projected systems and differential inclusions. System and Control Letters, 55:45-51, 2006.

10. B. Brogliato and W.P.M.H. Heemels. Observer design for Lur'e systems with multivalued mappings: a passivity approach IEEE Transactions on Automatic Control, 54(8):1996-2001, 2009.

11. M.K. Camlibel, W.P.M.H. Heemels, and J.M. Schumacher. On linear passive complementarity systems. European Journal of Control, 8:220-237, 2002.

12. M.K. Camlibel, W.P.M.H. Heemels, A.J. van der Schaft, and J.M. Schumacher. Switched networks and complementarity. IEEE Trans. Circuits Systems-I, 50:1036-1046, 2003.

13. R.W. Cottle, J.-S. Pang, and R.E. Stone. The Linear Complementarity Problem. Academic Press, Boston, 1992.

14. B. De Schutter and T. van den Boom. On model predictive control for max-min-plus-scaling discrete event systems. Automatica, 37(7):1049-1056, 2001.

15. P. Dupuis and A. Nagurney. Dynamical systems and variational inequalities. Annals of Operations Research, 44:9-42, 1993.

16. X. Fan and M. Arcak. Observer design for systems with multivariable monotone nonlinearities. Systems and Control Letters, 50:319-330, 2003.

17. R. Goebel, R. Sanfelice, and A.R. Teel. Hybrid dynamical systems. IEEE Control Systems Magazine, 29(2):28-93, 2009.

18. R. Goebel and A.R. Teel. Solution to hybrid inclusions via set and graphical convergence with stability theory applications. Automatica, 42:573-587, 2006

19. M.L.J. Hautus and L.M. Silverman. System structure and singular control. Linear Algebra and its Applications, 50:369402, 1983.

20. W.P.M.H. Heemels, M.K. Camlibel, B. Brogliato, and J.M. Schumacher. Observer-based control of linear complementarity systems. In M. Egerstedt and B. Mishra, editors, Proc. 11th International Workshop on Hybrid Systems: Computation and Control 2008 (St. Louis, USA), LNCS 4981, pages 259-272. Springer, 2008.

21. W.P.M.H. Heemels, M.K. Camlibel, and J.M. Schumacher. On the dynamic analysis of piecewise linear networks. IEEE Transactions on Circuits and Systems I, 49:315-327, 2002.

22. W.P.M.H. Heemels, M.K. Camlibel, A.J. van der Schaft, and J.M. Schumacher. Modelling, well-posedness, and stability of switched electrical networks. In Proc. 6th Intern. Workshop on Hybrid Systems: Computation and Control (Prague), volume 2623 of Lecture Notes in Computer Science, pages 249-266. Springer, 2003.

23. W.P.M.H. Heemels, B. De Schutter, and A. Bemporad. Equivalence of hybrid dynamical models. Automatica, 37(7):10851091, 2001.

24. W.P.M.H. Heemels, J. M. Schumacher, and S. Weiland. The rational complementarity problem. Linear Algebra and its Applications, 294:93-135, 1999. 
25. W.P.M.H. Heemels, J.M. Schumacher, and S. Weiland. Linear complementarity systems. SIAM Journal on Applied Mathematics, 60:1234-1269, 2000.

26. W.P.M.H. Heemels, J.M. Schumacher, and S. Weiland. Projected dynamical systems in a complementarity formalism. Operations Research Letters, 27(2):83-91, 2000.

27. G. Iulia Bara, J. Daafouz, F. Kratz, and C. Iung. State estimation for a class of hybrid systems. In Int. Conf. Automation of Mixed Processes, pages 313-316, 2000.

28. M. Johansson. Piecewise Linear Control Systems. Springer-Verlag, 2003.

29. A.Lj. Juloski, W.P.M.H. Heemels, and S. Weiland. Observer design for a class of piecewise linear systems. Intern. J. Robust and Nonlinear Control, 17(15):1387-1404, 2007.

30. H. Kwakernaak and R. Sivan. Linear Optimal Control Systems. Wiley, New York, 1972.

31. R.I. Leine and N. van de Wouw. Stability and Convergence of Mechanical Systems with Unilateral Constraints, volume 36 of Lecture Notes in Applied and Computational Mechanics. Berlin Heidelberg New-York, Springer-Verlag, 2008.

32. J. Lygeros, K.H. Johansson, S.N. Simic, J. Zhang, and S.S. Sastry. Dynamical properties of hybrid automata. IEEE Trans. Aut. Control, 48(1), 2003.

33. N. Lynch, R. Segala, F.W. Vaandrager, and H.B. Weinberg. Hybrid I/O automata. In Proc. Workshop on Hybrid Systems: Computation and Control, volume 1066 of LNCS, pages 496-510. Springer-Verlag, 1996.

34. M.D.P. Monteiro Marques. Differential Inclusions in Nonsmooth Mechanical Problems. Shocks and Dry Friction. Progress in Nonlinear Differential Equations and Their Applications 9. Birkhauser, Basel, 1993.

35. J.J. Moreau. Evolution problem associated with a moving convex set in a hilbert space. Journal of Differential Equations, 26:347-374, 1977

36. A. Nagurney and D. Zhang. Projected Dynamical Systems and Variational Inequalities with Applications. Kluwer, Dordrecht, 1996.

37. M. Osorio and J.A. Moreno. Dissipative design of observers for multivalued nonlinear systems. In Proc. CDC, pages 5400-5405, 2006

38. A. Pavlov, N. van de Wouw, and H. Nijmeijer. Convergent piecewise affine systems: analysis and design part i: continuous case. In Proc. CDC/ECC, Sevilla, Spain, 2005.

39. S. Petterson. Switched state jump observers for switched systems. In Proceedings of the IFAC World Congress, Prague, Czech Republic, 2005.

40. R. Rajamani. Observers for Lipschitz nonlinear systems. IEEE Trans. Aut. Control, 43:397-401, 1998.

41. R. G. Sanfelice, R. Goebel, and A. R. Teel. Invariance principles for hybrid systems with connections to detectability and asymptotic stability. IEEE Trans. Automat. Contr., 52(12):22822297, 2007.

42. J. Shen and J.S. Pang. Linear complementarity systems: Zeno states. SIAM Journal on Control and Optimization, 44:10401066,2005

43. J. Shen and J.S. Pang. Linear complementarity systems with singleton properties: non-Zenoness. In Proc. 2007 American Control Conference, pages 2769-2774, 2007

44. J. Shen and J.S. Pang. Semicopositive linear complementarity systems. Intern. J. Robust and Nonlinear Control, 17(15):1367-1386, 2007.

45. E.D. Sontag. Nonlinear regulation: The piecewise linear approach. IEEE Trans. Automatic Control, 26(2):346-358, 1981.

46. D. E. Stewart. Uniqueness for solutions of differential complementarity problems. Mathematical Programming Series A, 118:327-345, 2009.

47. N. van de Wouw and A. Pavlov. Tracking and synchronisation for a class of PWA systems. Automatica, 44(11):2909-2915, 2008.

48. A. J. van der Schaft and J. M. Schumacher. The complementary-slackness class of hybrid systems. Mathematics of Control, Signals and Systems, 9:266-301, 1996.

49. A.J. van der Schaft and J.M. Schumacher. Complementarity modeling of hybrid systems. IEEE Transactions on Automatic Control, 43:483-490, 1998.

50. F. Vasca, L. Iannelli, M.K. Camlibel, and R. Frasca. A new perspective for modeling power elctronics converters: complementarity framework. IEEE Transactions on Power Electronics, 24(2):456-468, 2009.

51. J.C. Willems. Dissipative dynamical systems. Archive for Rational Mechanics and Analysis, 45:321-393, 1972. 